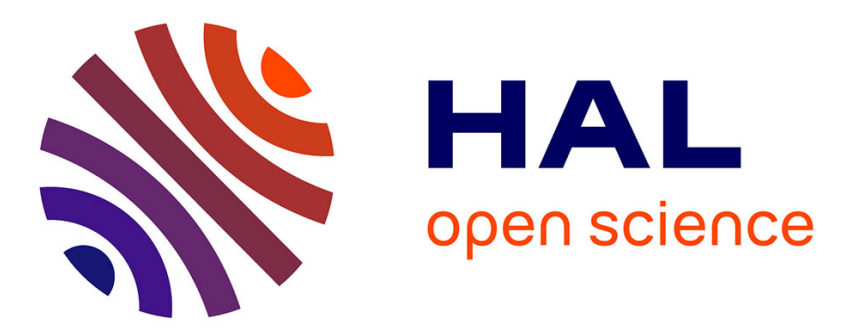

\title{
A zonal Galerkin-free POD model for incompressible flows
}

Michel Bergmann, Andrea Ferrero, Angelo Iollo, Edoardo Lombardi, Angela Scardigli, Haysam Telib

\section{- To cite this version:}

Michel Bergmann, Andrea Ferrero, Angelo Iollo, Edoardo Lombardi, Angela Scardigli, et al.. A zonal Galerkin-free POD model for incompressible flows. Journal of Computational Physics, 2018, 352, pp.301 - 325. 10.1016/j.jcp.2017.10.001 . hal-01668546

\section{HAL Id: hal-01668546 https://hal.inria.fr/hal-01668546}

Submitted on 9 Oct 2018

HAL is a multi-disciplinary open access archive for the deposit and dissemination of scientific research documents, whether they are published or not. The documents may come from teaching and research institutions in France or abroad, or from public or private research centers.
L'archive ouverte pluridisciplinaire HAL, est destinée au dépôt et à la diffusion de documents scientifiques de niveau recherche, publiés ou non, émanant des établissements d'enseignement et de recherche français ou étrangers, des laboratoires publics ou privés. 


\title{
A zonal Galerkin-free POD model for incompressible flows
}

\author{
Michel Bergmann ${ }^{1,2,3}$, Andrea Ferrero*1,2,3 Angelo Iollo $^{1,2,3}$, Edoardo Lombardi ${ }^{4}$, \\ Angela Scardigli, ${ }^{4,5}$, and Haysam Telib ${ }^{4}$ \\ ${ }^{1}$ Memphis Team, INRIA, F-33400 Talence, France \\ ${ }^{2}$ Univ. Bordeaux, IMB, UMR 5251, F-33400 Talence, France \\ ${ }^{3}$ CNRS, IMB, UMR 5251, F-33400 Talence, France \\ ${ }^{4}$ Optimad Engineering, 10143 Torino, Italy \\ ${ }^{5}$ Politecnico di Torino, 10129 Torino, Italy
}

\begin{abstract}
A domain decomposition method which couples a high and a low-fidelity model is proposed to reduce the computational cost of a flow simulation. This approach requires to solve the high-fidelity model in a small portion of the computational domain while the external field is described by a Galerkin-free Proper Orthogonal Decomposition (POD) model. We propose an error indicator to determine the extent of the interior domain and to perform an optimal coupling between the two models. This zonal approach can be used to study multi-body configurations or to perform detailed local analyses in the framework of shape optimisation problems. The efficiency of the method to perform predictive low-cost simulations is investigated for an unsteady flow and for an aerodynamic shape optimisation problem.
\end{abstract}

Keywords - POD, Reduced Order Model, boundary conditions, shape optimisation, Galerkin-free model

\section{Introduction}

One of the main issues in the simulation of fluid flows is the definition of appropriate boundary conditions. In most cases, the dilemma of the boundary conditions dictates de-facto the choice of the computational domain. Generally speaking, the computational domain is extended from the region of interest until its boundaries fall in a region for which some values or model for the boundary condition may be derived. Unfortunately, even when relaxing on the position of the boundary condition, exact information regarding the values are unknown in most cases. The dominant approach consists in trying to minimise the spurious effects of the boundary conditions by locating them as far as possible from the crucial region or at some natural choices (like sonic throats in nozzles). In fact, one of the crucial (empiric) engineering know-how, is to evaluate the trade-off between size of the computational domain and accuracy of the boundary conditions and how this choice impacts on the over-all design work-flow. A systematic approach to this problem is still out of reach for most industries, since error-bounds and uncertainties should be propagated and quantified throughout the entire design process.

In external aerodynamics the computed body forces are particularly sensitive to the location of the far-field boundaries where undisturbed values are assumed. In order to reduce the computational domain, two different families of approaches can be found in literature. A first set of family focuses on the implementation of absorbing and non-reflecting boundary conditions for both compressible and incompressible flows (see for example [41] and [29]). The main idea behind these methods is to allow the correct propagation of starting waves, which are generated at the body, out of the computational domain as if the domain was unbounded. The use of these boundary conditions introduces several benefits but the requirements on the size of the computational domain can still be demanding. For example, the distance between a lifting body and the far-field boundary modelled with non-reflecting boundary conditions is

*Corresponding author: andrea.ferrero@inria.fr 
up to hundreds times the characteristic size of the body [11].

Another family uses simplified flow models to derive boundary conditions closer to the solid. These techniques rely on the observation, that non-linear, viscous or rotational effects decay far from the body and that a simpler model may express the boundary conditions. Such an approach was proposed by Thomas and Salas [52] for flows around lifting bodies through a point vortex correction method. In their approach, the velocity boundary condition is improved by introducing a correction given by the velocity induced by a point vortex located on the body, whose intensity is derived by monitoring the circulation around the body. In other words, the knowledge of the computed flow field inside the domain is used to correct and improve automatically the boundary condition. In this way, the size of the computational domain can be significantly reduced without introducing a significant dependence of the solution on the shape of the external boundary. A similar approach has been proposed for the continuation of inviscid vortex patches in 2D configurations [22].

For internal flows, inlet and outlet conditions may be even harder to derive if the simulated component is part of large complex system. For these cases, multi-scale methods, which introduce a two-way coupling between the component (modelled through an high-fidelity model) and the system (modelled through a network of lumped coefficients) have proven to be a viable approach (see for example [21]). The two-way coupling is achieved through the boundary conditions, computed by the network model and used for the high-fidelity computation, and the lumped coefficients, computed by the high-fidelity model and used in the network model. The cost of re-iterations introduced by the two-way coupling is generally much lower than using the high-fidelity model throughout the entire system. For example, the performances of aerospace propulsion systems are usually evaluated by using this approach [53]. Another application concerns the simulation of the heart and its interaction with the circulatory system [42].

For the sake of the later discussion, we can say that boundary conditions are (sub) models, which may predict the values of flow variables at the boundary. The errors introduced depend on the position of the boundary and on the sophistication of the model. The easiest boundary conditions impose just a value, while more complicated boundary conditions may mimic an entire system.

Lately, empirical Reduced Order Models (ROM) are gaining considerable attention as a generic framework for reducing the computational cost of complex simulations. Most ROMs rely on an offline phase where numerical experiments are performed in order to collect the empirical knowledge necessary to derive the ROM. Within an online phase, the ROMs may be evaluated at a very competitive cost, which is generally orders of magnitude less than the original Full Order Model (FOM). Several ROM approaches are nowadays available: Proper Orthogonal Decomposition (POD) [13, 15, 26, 36, 45, 46, 49, 50, 59]), Proper Generalised Decomposition (PGD) [19], Reduced Basis (RB) [43, 44], Dynamic Mode Decomposition [47], Empirical Interpolation Method (EIM) [7], Discrete Empirical Interpolation Method (DEIM) [18], hierarchical model reduction [40].

Proper Orthogonal Decomposition is an attractive choice to define the reduced solution space. The POD algorithm allows to identify the most relevant structures in the flow field starting from a collection of snapshots [50]. This allows a significant compression of the information because just a few modes can contain the information, which is associated to several snapshots, especially in unsteady problems.

In the classical POD approach the equations which govern the flow field are projected onto the POD basis by means of a Galerkin projection [8, 36, 46]. This makes it possible to get a set of ordinary differential equations which describe the time evolution of the POD coefficients. A typical application of this approach is the study of flow control problems $[37,56,57]$. This approach is very powerful since it reduces significantly the number of degrees of freedom of the discretisation. However, the POD-Galerkin method can give unstable results, especially for high Reynolds number flows because it misses the stabilisation effects related to the truncated small scales. The classical remedy for this problem is based on the introduction of artificial dissipation terms which make the model stable [5, 8, 38, 55, 58]. Alternative techniques based on Petrov-Galerkin projections are also possible [17, 25]. All these approaches are characterised by a strong reduction in the number of degrees of freedom of the discretisation but their application to the prediction of general non-linear problems is challenging because they often require problem-dependent stabilisation terms.

A further burden of these approaches, especially in an industrial context, is the cost of the offline phase, which is often not discussed. The number of numerical realisations in order to have a representative 
database is strongly dependent on the size of the parameter space and on the non-linearity of the problem. Since POD modes are linear combinations of snapshots, non-linear behaviour could be recovered by sufficiently fine-grained sampling of the parameter space or by the use of local models based on machine learning techniques $[3,60]$. For example, in aerodynamic shape optimisation $\mathrm{O}(10)-\mathrm{O}(100)$ parameters are generally used and very non-linear effects like flow separation, recirculation bubbles, shocks and turbulence are the rule rather than the exception. In these cases, the cost of the generation of the requested database may not be amortised by the subsequent cost savings due to the ROM.

A further obstacle in projection based ROMs is the difficulty to account for geometrical modifications of the computational domain. Finite-element solvers can account for different element mappings if the connectivity vector remains unaltered throughout the database. If re-meshing of the domain is necessary or if topological changes are present, it may be very difficult or impossible to employ these methods.

In this work, a zonal Galerkin-free POD method is derived through a domain decomposition approach in which an high-fidelity solver is applied in a subset of the computational domain while the solution in the external region is described by a POD reconstruction. The core of this hybrid approach is a technique to derive near-field boundary conditions for the high-fidelity solver. This methodology can be seen as a generalisation of the point vortex correction method: instead of introducing a boundary correction based on one mode whose shape is decided a-priori (the point vortex), a general set of correction modes (the POD modes) is automatically built from an empirical basis. The POD reconstruction is then dynamically coupled to the high-fidelity solution by introducing some compatibility conditions in a weak sense. This methodology is not restricted to any class of problems, and may be adopted for simulations of compressible and incompressible flows of external and internal problems. Anyhow the expected computational gains may vary significantly depending on the considered problem.

Although this zonal approach gives a less impressive reduction of the computational cost with respect to the POD-Galerkin approach, this methodology may be a more suitable alternative for an industrial context. The method does not suffer from instability when non-linear phenomena are present and it does not need a priori a very fine sampling in order to predict them correctly. Since the method uses the canonical high-fidelity solver where the POD basis fails to reproduce non-linear effects, it simply exempts the reduced basis from this responsibility. For non-linear problems, it will be shown in the test cases considered in this paper that the sampling influences the size of the domain where the high-fidelity solver must be employed. Consequently the method will benefit from a finer sampling, since the high-fidelity domain may be further reduced while keeping error-bounds constant. Furthermore the method does not rely on any one-to-one mapping of the computational domain. Mesh and even topological changes in the geometry can be handled in a straight-forward manner.

While the basic idea of this approach has been proposed by Buffoni et al [14], in this work we present how to exploit the empirical basis in order to decide the domain-decomposition and how to derive an optimal coupling between the POD reconstruction and the high-fidelity solver. Furthermore the methodology is extended to incompressible flows.

The paper is outlined as follows. In section 2, in order to demonstrate that this approach is compatible with virtually any CFD tool, two solvers are presented, which have been enhanced with the proposed technology. In section 3, the zonal Galerkin-free POD approach is depicted. In section 4.1, a prediction error indicator is presented and employed to define the domain decomposition and to perform an optimal coupling between the high-fidelity solver and the POD reconstruction. In section 5, the performance of this methodology is evaluated by performing a study on the interaction between a vortex and a NACA0012 airfoil at low Reynolds numbers. In section 6, the methodology is applied in the framework of shape optimisation of an automotive test case in order to reduce aerodynamic drag solving the Reynolds Averaged Navier Stokes (RANS) equations. A critical discussion of the methodology, concluding remarks and an outline of further improvements are presented in a final section. 


\section{High-fidelity discretisation of incompressible Navier-Stokes equations}

The flow fields studied in this work are described by the incompressible Navier-Stokes equations (NS) or by the incompressible Reynolds Averaged Navier-Stokes (RANS) equations. The NS equations are:

$$
\begin{aligned}
& \frac{\partial \mathbf{u}}{\partial t}+(\mathbf{u} \cdot \nabla) \mathbf{u}=-\frac{1}{\rho} \nabla p+\nu \nabla^{2} \mathbf{u}, \\
& \nabla \cdot \mathbf{u}=0,
\end{aligned}
$$

where $\mathbf{u}$ and $p$ are the velocity and pressure fields, and $\nu=\mu / \rho$ is the kinematic viscosity, with $\mu$ the dynamic viscosity and $\rho$ is the density. The RANS equations are obtained by applying an average operator on the previous equations and introducing a closure model. The RANS model used in this work is the Spalart-Allmaras [51] one equation model.

The simulations reported in this work are performed with two different numerical models in order to test the zonal Galerkin-free POD approach with different numerical schemes and physical models. In particular, the simulations on the interaction between the airfoil and the vortex are performed by means of an immersed boundary cartesian code. The simulations related to the automotive shape optimisation problem are performed through the OpenFoam package[28].

\subsection{Projection scheme for low Reynolds number unsteady flow}

The incompressible Navier-Stokes equations are solved by means of an incremental fractional step method based on the approach of Chorin-Temam [20]. The method requires to perform a prediction step by integrating the momentum equation with time step $\Delta t$ in order to get an approximate intermediate state:

$$
\frac{\mathbf{u}^{*}-\mathbf{u}^{n}}{\Delta t}=-\frac{1}{\rho} \nabla \bar{p}-[(\mathbf{u} \cdot \nabla) \mathbf{u}]^{n+\frac{1}{2}}+\nu \nabla^{2} \mathbf{u}^{n+\frac{1}{2}},
$$

where $\bar{p}$ is an approximation of the pressure field at time $n+\frac{1}{2}$, and the convection and diffusion terms at time $n+\frac{1}{2}$ are obtained with an Adams-Bashforth extrapolation. Usually we chose $\bar{p}=p^{n}$ and we have:

$$
\mathbf{u}^{*}=\mathbf{u}^{n}+\Delta t\left[-\frac{1}{\rho} \nabla p^{n}-\left(\frac{3}{2}\left(\mathbf{u}^{n} \cdot \nabla\right) \mathbf{u}^{n}-\frac{1}{2}\left(\mathbf{u}^{n-1} \cdot \nabla\right) \mathbf{u}^{n-1}\right)+\nu\left(\frac{3}{2} \nabla^{2} \mathbf{u}^{n}-\frac{1}{2} \nabla^{2} \mathbf{u}^{n-1}\right)\right] .
$$

The space discretisation is based on finite differences evaluated on cartesian meshes with spacing $h$ for cell centered collocated variables. The convective terms are approximated by a third order upwind scheme. The diffusive terms are approximated by a second order centered scheme.

The intermediate velocity field $\mathbf{u}^{*}$ is not divergence-free. It is corrected by solving a Poisson problem on the pressure correction variable $\hat{\Phi}$ which makes it possible to get a divergence free velocity field at the end of the time step $\left(\mathbf{u}^{n+1}\right)$ :

$$
\begin{gathered}
\hat{\Phi}=\Delta t\left(p^{n+1}-p^{n}\right) . \\
\nabla^{2} \hat{\Phi}=\rho \nabla \cdot \mathbf{u}_{f}^{*} . \\
\mathbf{u}^{n+1}=\mathbf{u}^{*}-\frac{1}{\rho} \nabla \hat{\Phi},
\end{gathered}
$$

where $\mathbf{u}_{f}^{*}$ is the face centered velocity field built from cell centered velocities $\mathbf{u}^{*}[33]$,

$$
\begin{gathered}
\mathbf{u}^{* *}=\mathbf{u}^{*}+\left.\frac{\Delta t}{\rho} \nabla p^{n}\right|_{c c}, \\
\mathbf{u}_{f}^{*}=\mathcal{I}\left(\mathbf{u}^{* *}\right)-\left.\frac{\Delta t}{\rho} \nabla p^{n}\right|_{f c},
\end{gathered}
$$


where $\mathcal{I}$ denotes a function of interpolation from cell centered to face centered position. The subscripts $c c$ and $f c$ denote cell centered (over $2 h$ ) and face centered derivatives (over $h$ ), respectively. The use of $\mathbf{u}_{f}^{*}$ helps removing the possible odd-even oscillations.

Appropriate boundary conditions are imposed on $\mathbf{u}^{*}$ and $\hat{\Phi}$ to solve (3) and (5).

The presence of bodies immersed into the fluid is taken into account by a penalization term $\gamma \chi\left(\hat{\mathbf{u}}-\mathbf{u}^{*}\right)$ which is added to the right hand side of (3). The characteristic function is $\chi=1$ inside the body and $\chi=0$ elsewhere, $\gamma \gg 0$ is the penalization parameter and $\hat{\mathbf{u}}$ is the body velocity on nodes inside the body. In order to improve the accuracy in the wall region, the body velocity $\hat{\mathbf{u}}$ is artificially modified in the first layer of points inside the body to get a second order accurate velocity discretisation $[10,24]$.

\subsection{Steady simulation of RANS equations}

For the solution of the steady-state RANS equations, we use simpleFoam, an OpenFOAM solver based on a cell-centered finite volume method, implementing the SIMPLE pressure-velocity coupling proposed by Patankar [39]. As shown in [27], the method relies on the derivation of an explicit equation for the pressure, starting from the semi-discretised form of the momentum equation:

$$
a_{p} \mathbf{u}_{p}=\mathbf{H}(\mathbf{u})-\nabla p,
$$

where $a_{p}$ are the matrix diagonal coefficients and $\mathbf{H}(\mathbf{u})$ contains both the neighbours contributions and the source terms apart from the pressure gradient.

The continuity equation can be discretised in terms of the face fluxes as:

$$
\nabla \cdot \mathbf{u}=\sum_{f} \mathbf{s} \cdot \mathbf{u}_{f}=0,
$$

where $\mathbf{s}$ is the face area vector and $\mathbf{u}_{f}$ represents the velocity interpolated on the face, which is obtained from Eq. 7:

$$
\mathbf{u}_{f}=\left(\frac{\mathbf{H}(\mathbf{u})}{a_{c}}\right)_{f}-\left(\frac{1}{a_{p}}\right)_{f}(\nabla p)_{f} .
$$

Substituting Eq. 9 into Eq. 8, we obtain the discretised pressure equation:

$$
\sum_{f} \mathbf{s} \cdot\left[\left(\frac{1}{a_{p}}\right)_{f}(\nabla p)_{f}\right]=\sum_{f} \mathbf{s} \cdot\left(\frac{\mathbf{H}(\mathbf{u})}{a_{p}}\right)_{f} .
$$

Eq. 7, Eq. 8 and Eq.10 are solved iteratively through under-relaxation, until convergence is attained. First, the momentum equation is under-relaxed implicitly and solved in order to obtain the predicted velocity $\mathbf{u}^{\star}$, using the pressure field from the previous iteration to compute the pressure gradient term. $\mathbf{u}^{\star}$ is then used in the pressure equation to evaluate the new pressure distribution. Once that the new $p$ is obtained, the face fluxes are corrected and the new pressure field to be used in the momentum predictor step is computed by under-relaxing $p$.

The equations are discretised with Gaussian integration, using different schemes for each term. For the gradient terms, we use a second-order accurate centered scheme for $p$ and $\mathbf{u}$ and a cell limited version for turbulent quantities. The Laplacian terms are approximated by a blending of a bounded, first order, non-conservative scheme and an unbounded, second order, conservative scheme. The convective terms in the turbulence equations are discretised using a total variation diminishing (TVD) bounded scheme, whereas linear upwind differencing is employed in the momentum equation.

\section{A zonal Galerkin-free POD model}

The main idea is to solve the high-fidelity model where a given degree of accuracy is required and to approximate the solution by an empirical modal representation elsewhere. The objective is to reduce the extent of the domain where a high-fidelity simulation is performed, see Figure 1. 


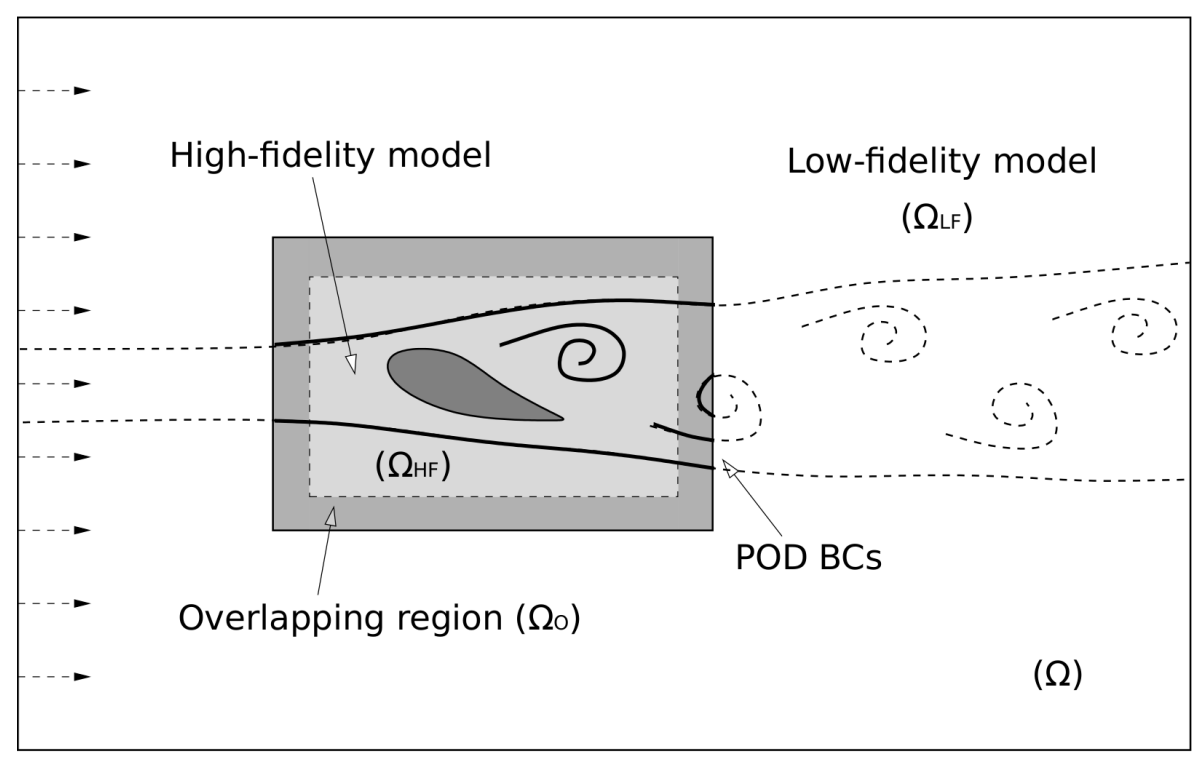

Figure 1: Zonal simulation based on domain decomposition.

The empirical low-dimensional representation is obtained by a previously computed database of highfidelity solutions on a large computational domain $(\Omega)$ for different working conditions or system configurations. This first step is performed offline. The sampling of the parameter space will strongly influence the accuracy of the modal representation and hence the ability to perform actual predictions (configurations not included in the database). In this sense, several techniques for an efficient sampling of the parameter space have been proposed in the literature (adaptive sampling for POD-base surrogates [54], adaptive sampling based on a leave-one-out error indicator [12, 60], compact POD bases [16], greedy approaches based on Voronoi tessellation [9]). Here, we will limit ourself to a simple cartesian sampling of the parameter space as explained in the following.

The whole computational domain $(\Omega)$ includes three regions: a domain $\left(\Omega_{H F}\right)$ where a high degree of accuracy is required, a domain $\left(\Omega_{L F}\right)$ where the solution is represented by empirical modes (here POD modes) and an overlapping region $\left(\Omega_{O}\right)$. We have:

$$
\Omega_{H F} \subset \Omega, \quad \Omega_{L F} \subseteq \Omega \quad \text { and } \quad \Omega_{O}=\Omega_{H F} \cap \Omega_{L F} .
$$

The high-fidelity solution and the low-order representation are matched in the overlapping domain $\Omega_{O}$ as explained in the following. Moreover, a criterium to determine $\Omega_{H F}$ based on an error indicator will be introduced in Section 4.2 .

\subsection{POD modal representation in $\Omega_{L F}$}

The zonal method described in this paper can be applied to different modal representations such as reduced basis $[43,44]$ or stability modes $[4,6,34,35]$ approaches. Here we focus on Proper Orthogonal Decomposition (POD) for the description of the external region.

The phenomena considered in the present study are described by the incompressible Navier-Stokes equations or by the incompressible Reynolds Averaged Navier-Stokes (RANS) equations. A modal description of the velocity field $\tilde{\mathbf{u}}$ and of the pressure field $\tilde{p}$ is: 


$$
\begin{array}{ll}
\tilde{\mathbf{u}}(\mathbf{x}, t)=\mathbf{f}_{\mathbf{u}}(\mathbf{x}, t)+\sum_{i=1}^{N} \hat{u}_{i}(t) \Phi_{\mathbf{i}}(\mathbf{x}) & \hat{u}_{i} \in \mathbb{R}, \Phi_{\mathbf{i}} \in \mathbb{R}^{d}, \mathbf{x} \in \Omega_{L F}, \\
\tilde{p}(\mathbf{x}, t)=f_{p}(\mathbf{x}, t)+\sum_{i=1}^{N} \hat{p}_{i}(t) \Psi_{i}(\mathbf{x}) & \hat{p}_{i} \in \mathbb{R}, \Psi_{i} \in \mathbb{R}, \mathbf{x} \in \Omega_{L F},
\end{array}
$$

where $N$ is the number of modes, $d$ is the space dimension and $\boldsymbol{\Phi}_{i}$ and $\Psi_{i}$ are the velocity and pressure modes, respectively. The variable $\mathbf{x}$ identifies the position in space and the parameter $t$ represents the time if unsteady problems are considered. In steady problems, the parameter $t$ can be seen as a parameter which identifies the working condition or the configuration of the system.

In some applications it is useful to set the forcing fields $\left(\mathbf{f}_{\mathbf{u}}\right.$ and $f_{p}$ ) equal to the average fields obtained by applying an average operator to all the configurations in the database. For example, in external aerodynamic problems the average velocity field is a key quantity because it fixes the far field velocity magnitude.

According to the approach of Sirovich [50] the POD modes $\left(\boldsymbol{\Phi}_{i}, \Psi_{i}\right)$ can be computed as a linear combination of $N_{s}$ snapshots $\left(\mathbf{u}_{j}(\mathbf{x})=\mathbf{u}\left(\mathbf{x}, t_{j}\right), p_{j}=p\left(\mathbf{x}, t_{j}\right)\right)$. Here, to fix notations, we briefly detail the procedure for the velocity field but it can be applied exactly in the same way for any other variable. In this work, the POD modes describe the difference between $\tilde{\mathbf{u}}$ and $\mathbf{f}_{\mathbf{u}}$, hence we have

$$
\boldsymbol{\Phi}_{i}(\mathbf{x})=\sum_{j=1}^{N_{s}} b_{j}\left(\mathbf{u}_{j}(\mathbf{x})-\mathbf{f}_{\mathbf{u}}\left(\mathbf{x}, t_{j}\right)\right)
$$

Let define the discrete scalar product operator $\langle\cdot, \cdot\rangle_{\Omega_{L F}}$ according to the numerical scheme which is used in the high-fidelity model. In this work a finite volume (FV) scheme on unstructured meshes or a finite difference (FD) scheme on uniform cartesian meshes are used and hence we have

$$
\langle\mathbf{g}(\mathbf{x}), \mathbf{h}(\mathbf{x})\rangle_{\Omega_{L F}}=\int_{\Omega_{L F}} \mathbf{g}(\mathbf{x}) \cdot \mathbf{h}(\mathbf{x}) d \Omega \approx \sum_{l=1}^{N_{p}} \mathbf{g}\left(\mathbf{x}_{l}\right) \cdot \mathbf{h}\left(\mathbf{x}_{l}\right) V_{l},
$$

where $N_{p}$ is the number of degrees of freedom used by the numerical scheme to describe the solution in $\Omega_{L F}$ and $V_{l}$ is the volume related to the $l^{\text {th }}$ cell of the discretisation. Let $\|\cdot\|_{\Omega_{L F}}$ be the norm related to this scalar product.

The procedure for computing the coefficients $b_{j}$ is based on a maximisation problem. The POD modes are found such that the projection of the snapshots on the modes is maximised:

$$
\max _{b_{j}}\left[\sum_{k=1}^{N_{s}}\left\langle\mathbf{u}_{k}(\mathbf{x})-\mathbf{f}_{\mathbf{u}}\left(\mathbf{x}, t_{k}\right), \mathbf{\Phi}_{i}(\mathbf{x})\right\rangle_{\Omega_{L F}}^{2}\right]=0 \quad \text { subject to }\left\|\mathbf{\Phi}_{i}(\mathbf{x})\right\|_{\Omega_{L F}}=1 \quad 1 \leq i, j \leq N_{s} .
$$

When Eq. 13 is substituted in Eq. 15 an eigenvalue problem is obtained. The eigenvectors of the resulting system give the coefficients $b_{j}$ required by Eq. 13. The associated eigenvalues rank the contribution of each POD mode to the representation of the snapshot database.

In this work two completely independent POD basis are built for the velocity and pressure fields.

The approach described here for pressure and velocity is applied exactly in the same manner also to the variables of the turbulence model when RANS equations are considered.

The eigenvalues $\left(\lambda_{i}\right)$ related to the POD modes allow to estimate the contribution given by each mode to the reconstruction of the snapshots. The sum of all the eigenvalues can be seen as the Total Information (energy) Content related to the fluctuations in the snapshots. The Relative Information Content $(R I C)$ indicator can be defined as:

$$
R I C(N)=\frac{\sum_{i=1}^{N} \lambda_{i}}{\sum_{i=1}^{N_{s}} \lambda_{i}} \quad 0 \leq N \leq N_{s},
$$


where $N_{s}$ is the number of snapshots and hence the maximum possible size of the POD basis.

Assuming that the eigenvalues are ordered with decreasing value, it is possible to choose the size $(N)$ of the POD basis by truncating the expansion after the first term which guarantees a given level of information $R I C>\sigma_{E}$ (for example 99.9\%).

\subsection{Galerkin-free POD model}

In $\Omega_{O}$ both the high-fidelity and the POD modal representation are defined. This region is the key to perform the coupling between the two models in the zonal simulation. On one hand, the POD solution is used to define the boundary conditions required by the high-fidelity solver. On the other hand, the high-fidelity solution inside the overlapping region is used to evaluate the POD expansion coefficients $\left(\hat{u}_{i}\right.$ and $\hat{p}_{i}$ ) which appear in Eq.12. This introduces a feedback of the high-fidelity solution onto the POD reconstructin which will be updated at each time step according to the evolution of the high-fidelity solution.

In order to compute the coefficients of the POD expansion, a minimisation problem is defined. In particular, the distance between the POD reconstruction $(\tilde{\mathbf{u}}, \tilde{p})$ and the high-fidelity solution $(\mathbf{u}, p)$ is minimised in the L2-norm sense in the overlapping region, in order to match the two representations:

$$
\begin{gathered}
\left\{\hat{u}_{i}\right\}_{i=1}^{N}=\operatorname{argmin}\left[\langle\mathbf{u}(\mathbf{x}, t)-\tilde{\mathbf{u}}(\mathbf{x}, t), \mathbf{u}(\mathbf{x}, t)-\tilde{\mathbf{u}}(\mathbf{x}, t)\rangle_{\Omega_{O}}\right] . \\
\left\{\hat{p}_{i}\right\}_{i=1}^{N}=\operatorname{argmin}\left[\langle p(\mathbf{x}, t)-\tilde{p}(\mathbf{x}, t), p(\mathbf{x}, t)-\tilde{p}(\mathbf{x}, t)\rangle_{\Omega_{O}}\right] .
\end{gathered}
$$

This leads to the definition of a small linear system ( $\operatorname{size} N$ ) whose solution gives the coefficients of the expansion $\left(\hat{u}_{i}, \hat{p}_{i}\right)$ which match the POD and the high-fidelity solutions at the current iteration of the zonal simulation:

$$
\begin{array}{ll}
\sum_{i=1}^{N} \hat{u}_{i}(t)\left\langle\boldsymbol{\Phi}_{i}(\mathbf{x}), \boldsymbol{\Phi}_{j}(\mathbf{x})\right\rangle_{\Omega_{O}}=\left\langle\mathbf{u}(\mathbf{x}, t)-\mathbf{f}_{\mathbf{u}}(\mathbf{x}, t), \boldsymbol{\Phi}_{j}(\mathbf{x})\right\rangle_{\Omega_{O}} & 1 \leq j \leq N . \\
\sum_{i=1}^{N} \hat{p}_{i}(t)\left\langle\Psi_{i}(\mathbf{x}), \Psi_{j}(\mathbf{x})\right\rangle_{\Omega_{O}}=\left\langle p(\mathbf{x}, t)-f_{p}(\mathbf{x}, t), \Psi_{j}(\mathbf{x})\right\rangle_{\Omega_{O}} & 1 \leq j \leq N .
\end{array}
$$

The overlapping region shown in Figure 1 consists in a layer of points close to the boundary of the high-fidelity domain. However, more general shapes can be considered. A possible criterium for the choice of the overlapping region is reported in Section 4.3.

\section{Definition of $\Omega_{H F}$ and $\Omega_{O}$}

\subsection{A prediction error indicator}

The zonal approach requires to split the computational domain in a region described by the high-fidelity solution and a region described by the POD reconstruction. However, it is not clear how to perform this splitting a priori. In order to answer this question an offline error indicator obtained from the snapshot database is proposed.

The main idea is to identify the regions where the representation of the flow field by the POD basis is strongly affected by a change in the input parameters. In order to find these zones we apply a leaveone-out strategy to the simulation database which contains the information from $N_{s p}$ sampling points, where a sampling point represents a configuration in the space of the parameters. This method requires to iteratively remove each sampling point from the database in turn and to use the remaining simulations to build a new POD basis. This new basis then is used to approximate the missing configuration and to get an insight on the ability of the basis to reconstruct new configurations.

The leave-one-out method belongs to the class of the cross-validation techniques used for model selection 
in statistics [23] and can be used as a procedure to perform adaptive sampling for building robust POD models $[12,60]$.

In this work, the leave-one-out strategy is not used to improve the sampling of the database but to get an error distribution map which can be exploited to choose the size of $\Omega_{H F}$ and $\Omega_{O}$.

In order to clarify the strategy, we apply this approach to the velocity field but the same analysis can be performed on any other variable which appears in the governing equations. Let the database include $N_{s p}$ sampling points and $N_{s}^{k}$ be the number of snapshots related to the $k^{t h}$ sampling point. This means that the number of snapshots in the database will be equal to $N_{s}=\sum_{k=1}^{N_{s p}} N_{s}^{k}$.

The $N_{s}^{k}$ snapshots of the $k^{t h}$ simulation (in the following denoted as $\mathbf{u}_{j}^{k}$ ) are removed from the database and the remaining snapshots are used to build a POD basis. In this offline phase, we set $\Omega_{L F}=\Omega$. The missing snapshots $\mathbf{u}_{j}^{k}$ are projected onto the POD basis in the domain $\Omega_{L F}$ :

$$
\mathcal{P}_{P O D}\left(\mathbf{u}_{j}^{k}(\mathbf{x})\right)=\mathbf{f}_{\mathbf{u}}\left(\mathbf{x}, t_{j}\right)+\sum_{i=1}^{N}\left\langle\mathbf{u}_{j}^{k}(\mathbf{x})-\mathbf{f}_{\mathbf{u}}\left(\mathbf{x}, t_{j}\right), \boldsymbol{\Phi}_{i}(\mathbf{x})\right\rangle_{\Omega_{L F}} \boldsymbol{\Phi}_{i}(\mathbf{x}) .
$$

The projection $\mathcal{P}_{P O D}\left(\mathbf{u}_{j}^{k}\right)$ is compared with the missing high-fidelity snapshot $\left(\mathbf{u}_{j}^{k}\right)$. The differences between these two fields are used to define an error indicator $\left(e_{j}^{k}(\mathbf{x})\right)$ which represents an estimate of the error that would be associated to the prediction of new configurations out of the database.

When the analysis is applied to the velocity field a possible approach is to define the error indicator as the error on the velocity magnitude $(|\mathbf{u}|=\sqrt{\mathbf{u} \cdot \mathbf{u}})$ :

$$
e_{j}^{k}(\mathbf{x})=|| \mathbf{u}_{j}^{k}(\mathbf{x})|-| \mathcal{P}_{P O D}\left(\mathbf{u}_{j}^{k}\right)(\mathbf{x})||
$$

The maximum of the reconstruction error $\left(e_{k}(\mathbf{x})\right)$ is computed over all the $N_{s}^{k}$ snapshots of the $k^{t h}$ simulation that was removed from the database. The same quantity is computed for all the $N_{s p}$ configurations in the database and finally the maximum between all the configurations is computed $(e(\mathbf{x}))$. The pseudocode reported in the Algorithm 1 summarises the method.

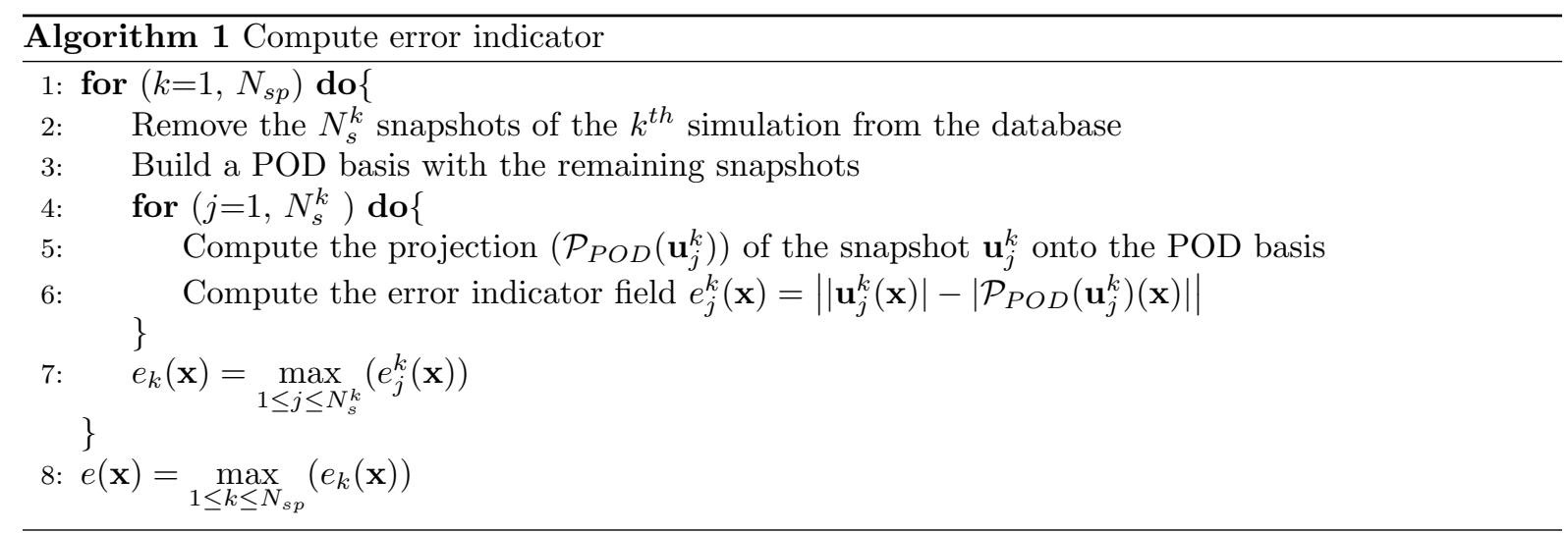

The procedure is simplified when steady problems are considered because in that case there is only one snapshot for each simulation $\left(N_{s}^{k}=1,1 \leq k \leq N_{s p}\right)$.

\subsection{Determination of $\Omega_{H F}$}

The error indicator field $e(\mathbf{x})$ obtained by the analysis of the database can be used to estimate the required extension of the high-fidelity domain $\Omega_{H F}$. In particular, the error indicator shows which are the zones where the solution is strongly influenced by the working conditions and where the POD reconstruction introduces large approximation errors. We propose to choose the size of $\Omega_{H F}$ by defining a threshold on the acceptable error $\sigma_{R}$. Ideally, $\Omega_{H F}$ should be chosen as the set of points in which the reconstruction error $e(\mathbf{x})$ is larger than the threshold $\sigma_{R}$ :

$$
\tilde{\Omega}_{H F}=\left\{\mathbf{x} \in \Omega: e(\mathbf{x})>\sigma_{R}\right\} .
$$


In this way, the regions where the POD model is not able to perform accurate predictions are directly studied by means of the high-fidelity solver. Practically, Eq.23 would lead to an irregular shape of the computational domain and so $\Omega_{H F}$ can be chosen as a rectangular box which includes $\tilde{\Omega}_{H F}$.

The extension of $\Omega_{H F}$ could become similar to the size of the full domain $\Omega$ for small values of the error threshold $\sigma_{R}$. In this case the computational gain of the zonal approach would vanish. The occurrence of such a situation would mean that the POD basis is not suitable to perform predictive simulations with the required accuracy. In other words, the database does not contain enough information and should be improved.

The error indicator field $e(\mathbf{x})$ obtained at the end of the leave-one-out procedure does not allow to identify which are the regions in the parameters space which require more exploration to improve the database. However, it is possible to compare the $N_{s p}$ different error fields $e_{k}(\mathbf{x})$ obtained during the leave-one-out analysis: if $e_{k}(\mathbf{x})$ is significantly larger for certain values of $k$ then it is useful to add new sampling points in the parameters space in the neighbouring of the $k^{t h}$ sampling point. An example of this strategy is adopted in [60].

\subsection{Determination of $\Omega_{O}$}

After choosing the size of $\Omega_{H F}$ it is necessary to define the overlapping region $\Omega_{O}$. The most natural choice would be to set the overlapping region equal to the entire high-fidelity region $\Omega_{H F}$. In this way, the coefficients of the POD expansion would be computed by minimising the distance between the highfidelity and the POD reconstruction in all the points of $\Omega_{H F}$. However, this choice could be suboptimal when performing predictive zonal simulations.

This aspect can be understood by considering the prediction error distribution obtained by the Algorithm 1. The error distribution shows that the regions where the reconstruction error is maximum are inside $\Omega_{H F}$. This means that there are some points inside $\Omega_{H F}$ in which the POD basis is not able to predict properly the solution of a new configuration. It could be useful to investigate how the inclusion of these points into the overlapping region will influence the prediction error on a given goal function. In particular, it is possible to exclude from the overlapping region all those points which are characterised by a prediction error larger than a given threshold $\left(\sigma_{O}\right)$. In other words, if the POD basis gives a very poor prediction in a region it could be better not to use that region for the computation of the POD coefficients. The previous idea can be exploited in the framework of a leave-one-out strategy to evaluate the effects of the choice of the overlapping region on the prediction error related to a given goal function. The procedure is described by the pseudocode reported in Algorithm 2 where $\sigma_{O}^{\min }, \sigma_{O}^{\max }$ and $\Delta \sigma_{O}$ represent the boundaries and the sampling size of the range in which $\sigma_{O}$ is investigated. This analysis can be done offline and requires $N_{s p} \frac{\sigma_{O}^{\max }-\sigma_{O}^{\min }}{\Delta \sigma_{O}}$ zonal POD simulations.

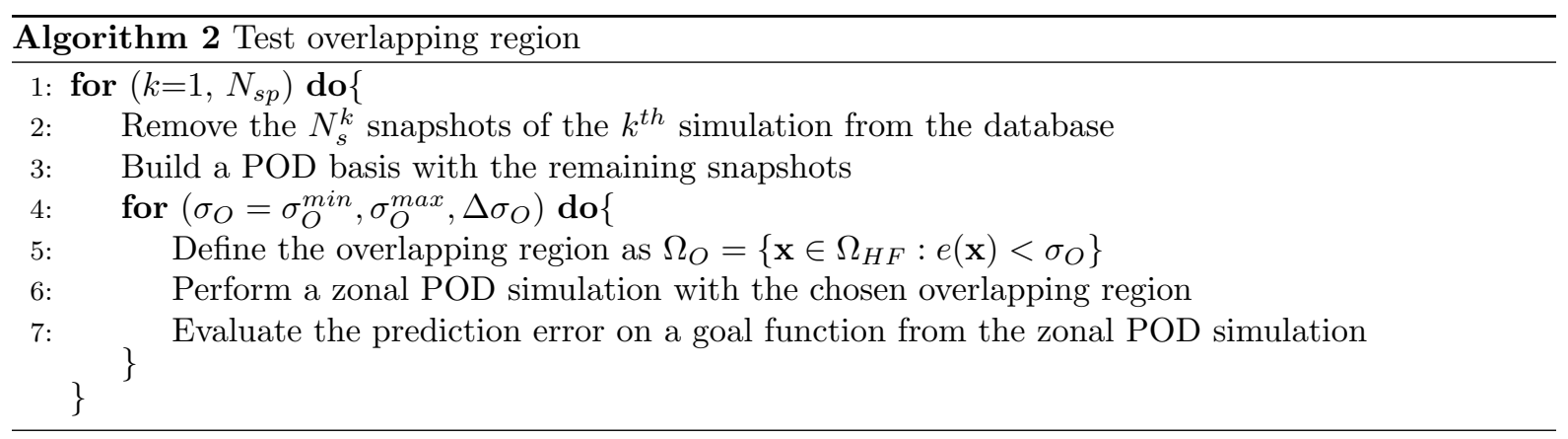

In general it is possible to perform the previous analysis for each state variable which appears in the physical model in order to define an ad-hoc overlapping region for each variable. Alternatively, it is possible to perform the study only on a single variable (for example the velocity) and then the same overlapping region can be used for all the variables (velocity, pressure and turbulence model's variables).

\subsection{Extension of $\Omega_{L F}$ and goal function}

The leave-one-out strategy described at the beginning of this Section is based on the use of a POD basis defined on the full computational domain $(\Omega)$. This is a natural choice that can be always done when 
Algorithm 1 is applied during the offline analysis.

As far as the zonal POD simulations are concerned, there are several possible choices about the extension of $\Omega_{L F}$. In particular, the extension of the zone in which the POD solution is needed depends on the goal function which is under study. Consider for example the configuration reported in Figure 1. If the goal is the prediction of the force on the airfoil then the zonal simulation can be performed by using a POD basis which is defined only in the high-fidelity region $\left(\Omega_{H F}\right)$ and in a layer of points on its boundary in order to define the boundary conditions required by the high-fidelity solver. This is due to the fact that the goal function can be directly computed by integrating the solution inside the high-fidelity domain $\left(\Omega_{H F}\right)$ and the knowledge of the external solution is useless for this purpose. In this way, the POD basis can be efficiently exploited to describe only the useful information and the energy ranking of the modes will not be affected by the structures in the external flow field. This means that less modes could be required to get a given accuracy level with respect to a POD basis defined everywhere. This approach will be used in the example of Section 5 .

On the other hand, there are configurations in which the goal function is an integral quantity which depends on both the internal high-fidelity solution and the external POD reconstruction. The problem studied in Section 6 is an example. In this case, the POD basis used for the predictive zonal POD simulations must be defined also in the external region $\left(\Omega \supset \Omega_{L F} \supset \Omega_{H F}\right)$.

\section{An unsteady laminar problem: interaction between a NACA0012 airfoil and a vortex}

In this Section the unsteady interaction between a NACA0012 airfoil and a vortex is studied. The vortex can be seen as a simplified model of gust. This example is used to test the ability of the zonal POD approach to perform low cost predictive simulations. In particular, some high-fidelity simulations are performed for different gust configurations and then the results are used to perform low cost predictions for new gust configurations.

The chosen working conditions are characterised by a Reynolds number based on the chord length equal to $R e=1000$ and an angle of attack $\alpha=5^{\circ}$. In this configuration, the flow field is laminar and steady. All the lengths and velocities which will appear in the following will be considered dimensionless and normalised with respect to the airfoil chord $(c)$ and the far field velocity magnitude $\left(U_{\infty}\right)$. The initial velocity field is given by the steady solution perturbed by the introduction of a vortex whose center is in $(-3,0)$. The magnitude of the velocity perturbation induced by the vortex is described by the following equation:

$$
q= \begin{cases}U_{0} \frac{r}{R} & \text { if } r<R \\ U_{0} \frac{R^{2}}{r^{2}} & \text { if } r \geq R\end{cases}
$$

where $q, r, R$ and $U_{0}$ are the magnitude of the induced velocity, the distance from the vortex center, the vortex characteristic radius and the vortex characteristic speed, respectively. The initial velocity magnitude field is reported in Figure 2.

The interaction between the vortex and the airfoil leads to a strong increase in the lift coefficient $\left(C_{l}\right)$ of the airfoil which is a key quantity in the design of a wing because it defines the load applied to the structures.

The high-fidelity simulations used to build the database are performed with the following setup. The computational domain $\Omega$ is chosen sufficiently large $(-8<x<8,-4<y<4)$ to include the vortex into the initial solution. The projection scheme described in Section 2.1 is used for the discretisation of the Navier-Stokes equations. As far as boundary conditions are concerned, the following choices are done for the velocity variable: Dirichlet boundary conditions are used for the inlet on the left side; symmetric boundary conditions are used on the lateral boundaries; non-reflecting boundary conditions [29] are used for the outlet on the right side. Homogeneus Neumann boundary conditions are imposed for pressure on all the boundaries.

A uniform cartesian mesh with $1600 \times 800$ points is used for the discretisation of $\Omega$. The mesh size is $\Delta x=\Delta y=0.01$ so there are 100 points on the chord of the airfoil. The lift coefficient for the undisturbed airfoil computed with the chosen mesh resolution $\left(C_{l}=0.25\right)$ is in good agreement with the 
results available in the literature $\left(C_{l}=0.25\right.$ according to [32]).

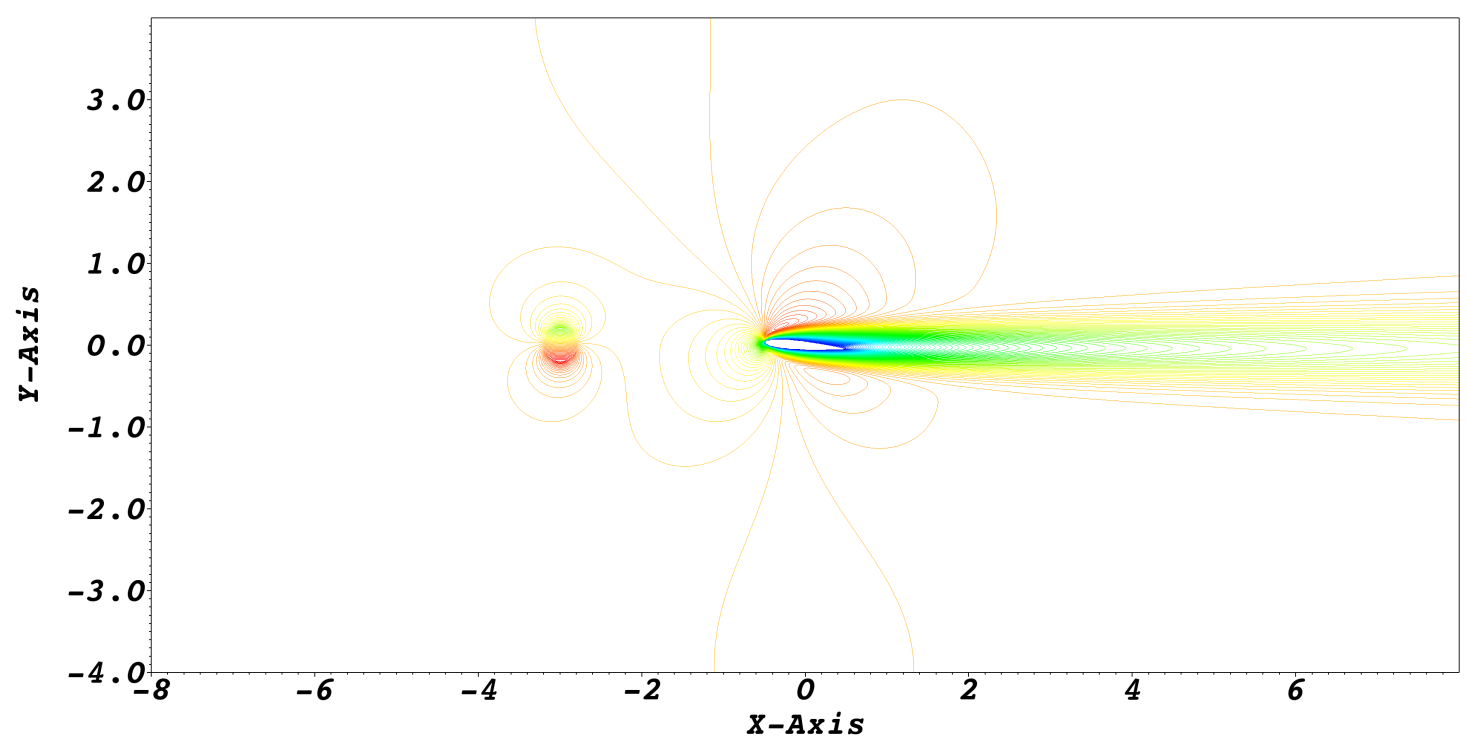

Figure 2: Initial condition for the NACA0012-vortex test case (velocity magnitude field).

\subsection{Forcing terms for zonal POD simulations with time-dependent BCs}

The procedure described in Section 3.2 makes it possible to update automatically the boundary conditions of the zonal POD simulation according to what is happening inside $\Omega_{H F}$. However, particular care should be taken in the definition of the forcing terms $\left(\mathbf{f}_{\mathbf{u}}, f_{p}\right)$ which appear in Eq.12 when dealing with problems characterised by time-dependent BCs.

Consider a body which is subjected to a time dependent perturbation in the incoming velocity, for example a gust or a vortex which interacts with the body. At the beginning of the simulation the gust is far from the body. The purpose of the zonal POD approach is to reduce the cost of the simulation by reducing $\Omega_{H F}$ to a region close to the body. However, this means that $\Omega_{H F}$ could not include the gust at the initial time step. According to Eqs. 17 and 18, the POD coefficients are computed by using the information which is present inside $\Omega_{H F}$ : if the gust is not inside $\Omega_{H F}$ (and so it is not inside $\Omega_{O}$ ), the coupling procedure will never activate the modes related to the gust and so the gust will never enter inside $\Omega_{H F}$.

In order to avoid this shortcoming, it is possible to augment the forcing terms with functions $\left(\mathbf{u}_{\mathbf{g}}\right.$ and $p_{g}$ ) which approximately describe the translation of the gust:

$$
\begin{aligned}
& \mathbf{f}_{\mathbf{u}}(\mathbf{x}, t)=\overline{\mathbf{u}}(\mathbf{x})+\mathbf{u}_{\mathbf{g}}(\mathbf{x}, t), \\
& f_{p}(\mathbf{x}, t)=\bar{p}(\mathbf{x})+p_{g}(\mathbf{x}, t),
\end{aligned}
$$

where the quantities $\overline{\mathbf{u}}(\mathbf{x})$ and $\bar{p}(\mathbf{x})$ represent the time averaged velocity and pressure fields obtained from a high-fidelity simulation without the gust.

The terms $\mathbf{u}_{\mathbf{g}}$ and $p_{\mathrm{g}}$ represent just a pure convection of the initial perturbation. They do not include any dissipative effect and they do not take into account the interaction between the gust and the body: these corrections will be introduced by the POD modes.

In other words, the POD modes will now be used to describe the difference between the real solution and an artificial solution in which the interaction between the gust and the body is neglected.

Thanks to this approach, it is possible to perform a zonal POD simulation even if the gust is not inside $\Omega_{H F}$ at the initial time: the time dependent forcing terms will automatically update the boundary conditions of the high-fidelity solver in order to force the gust to enter into $\Omega_{H F}$. 
Table 1: Convergence of the POD pressure modes w.r.t. the number of snapshots

\begin{tabular}{|c|c|c|c|}
\hline Mode $i$ & $\Delta_{20}^{40}\left(\Psi_{i}\right)$ & $\Delta_{40}^{80}\left(\Psi_{i}\right)$ & $\Delta_{80}^{160}\left(\Psi_{i}\right)$ \\
\hline 1 & $1.11 \mathrm{e}-02$ & $6.59 \mathrm{e}-04$ & $1.03 \mathrm{e}-04$ \\
\hline 2 & $3.41 \mathrm{e}-02$ & $4.23 \mathrm{e}-03$ & $3.00 \mathrm{e}-03$ \\
\hline 3 & $1.61 \mathrm{e}-01$ & $1.19 \mathrm{e}-02$ & $7.29 \mathrm{e}-03$ \\
\hline 4 & $2.00 \mathrm{e}-01$ & $1.65 \mathrm{e}-02$ & $8.46 \mathrm{e}-03$ \\
\hline 5 & $1.65 \mathrm{e}-01$ & $1.05 \mathrm{e}-02$ & $3.82 \mathrm{e}-03$ \\
\hline 6 & $1.43 \mathrm{e}-01$ & $5.86 \mathrm{e}-03$ & $2.12 \mathrm{e}-03$ \\
\hline 7 & $1.75 \mathrm{e}-01$ & $4.29 \mathrm{e}-03$ & $1.50 \mathrm{e}-03$ \\
\hline 8 & $2.47 \mathrm{e}-01$ & $4.98 \mathrm{e}-03$ & $2.76 \mathrm{e}-03$ \\
\hline 9 & $2.06 \mathrm{e}-01$ & $6.69 \mathrm{e}-03$ & $4.02 \mathrm{e}-03$ \\
\hline 10 & $4.21 \mathrm{e}-01$ & $8.60 \mathrm{e}-03$ & $2.20 \mathrm{e}-03$ \\
\hline 11 & $4.08 \mathrm{e}-01$ & $9.06 \mathrm{e}-03$ & $6.19 \mathrm{e}-03$ \\
\hline 12 & $5.74 \mathrm{e}-01$ & $1.09 \mathrm{e}-02$ & $3.07 \mathrm{e}-03$ \\
\hline
\end{tabular}

\subsection{Number of modes and number of snapshots}

First of all, a study on the convergence of the POD modes is performed in order to understand how many modes and how many snapshots are required. The attention is focused on a single simulation for a given vortex configuration and the flow field is uniformly sampled in time in order to have 20, 40, 80 and 160 snapshots. The snapshots are used to build POD bases defined on the full domain $\Omega$, so here we set $\Omega=\Omega_{L F}$. The eigenvalue analysis performed on the POD bases obtained with 20 and 160 snapshots shows that 12 modes are enough to describe $99 \%$ of the $R I C$ for both pressure and velocity (see Eq.16). In order to understand how many snapshots are required to assure the convergence of the first 12 POD modes the analysis presented in Table 1 is performed. In particular, four POD bases are built using 20, 40, 80 and 160 snapshots respectively. The quantity reported in the Table 1 represents the relative variation of the pressure mode $\left(\Psi_{i}\right)$ defined as

$$
\Delta_{n}^{m}\left(\Psi_{i}\right)=\frac{\sqrt{\left(\Psi_{i}^{n}-\Psi_{i}^{m}, \Psi_{i}^{n}-\Psi_{i}^{m}\right)_{\Omega_{L F}}}}{\left\|\Psi_{i}^{n}\right\|_{\Omega_{L F}}}=\sqrt{\left(\Psi_{i}^{n}-\Psi_{i}^{m}, \Psi_{i}^{n}-\Psi_{i}^{m}\right)_{\Omega_{L F}}},
$$

where $n$ and $m$ are the number of snapshots used to build the POD bases and the denominator was simplified thanks to the fact that all the modes have unit norm by definition. The data show that when the number of snapshots is increased the modes converge quickly. Similar results are obtained by checking the convergence of the velocity modes. According to these results, the following study will be performed by taking 130 snapshots from each simulation, i.e. $N_{s}^{k}=130,1 \leq k \leq N_{s p}$. The first four modes for the velocity magnitude field are shown in Figure 3.

\subsection{Analysis of the database: choice of the zonal setup}

Three different databases of high-fidelity simulations (with $N_{s p}=4,9,25$ in turn) are created by considering several values of vortex intensity and characteristic radius. In particular, a uniform sampling of the space of the parameters is chosen in the range $0.2 \leq U_{0} \leq 0.3$ and $0.2 \leq R \leq 0.4$. More efficient sampling techniques (see Section 3) could be used when the dimension of the parameter space is increased but the uniform sampling is sufficient for the purpose of this work. The sampling points belonging to the $2 \times 2$, $3 \times 3$ and $5 \times 5$ databases are shown in Figure 4 .

The time evolution of the lift coefficient for the high-fidelity simulations included in the $3 \times 3$ database is reported in Figure 5 showing its strong dependency on the chosen parameters. The time variable which appears in this plot and in the following ones is normalised with respect to the convection reference time obtained from the chord length and the far field velocity magnitude. 
The study presented in Section 5.2 showed that 130 snapshots contain enough information to properly describe the unsteady flow structures which characterise a single simulation. For this reason, in the following analysis 130 snapshots will be collected from each sampling point in the parameter space, i.e. $N_{s}^{k}=130,1 \leq k \leq N_{s p}$. The snapshots are pre-processed by removing the undisturbed steady field and the artificial forcing terms related to the considered gust configuration. The analysis of the $R I C$ (see Eq. 16) shows that 45 POD modes are sufficient to get $99 \%$ of the information in the database, for all the three considered databases. For this reason, in all the following simulations the size of the POD basis will be fixed to $N=45$.

The databases are analysed according to the leave-one-out strategy described in Section 4.2. The results are presented in Figure 6 which shows the prediction error indicator map for the velocity magnitude. The larger error values are localised close to the body (whose center is in $(0,0)$ ). The error map is defined everywhere, also inside the solid body, because the high-fidelity solver is based on a penalised immersed boundary approach in which the numerical solution is defined also inside the body. The results in Figure 6 show that when the database is enriched the maximum reconstruction error is reduced and the region where the error indicator is significant becomes smaller.

This last effect is also put in evidence in Figure 7 where the required number of mesh points in $\Omega_{H F}$ is reported as a function of $\sigma_{R}$. In particular, for each value of $\sigma_{R}$, the plot shows the number of mesh points required by the smallest rectangle which includes the region $\tilde{\Omega}_{H F}$ (see Eq.23). The number of mesh points is normalised with respect to the number of mesh points in $\Omega$ to give a direct evaluation of the reduction of the number of degrees of freedom. The plot shows that, for a given database, the size of $\Omega_{H F}$ must be increased if $\sigma_{R}$ is reduced. Furthermore, for a given threshold $\sigma_{R}$, the size of $\Omega_{H F}$ decreases when the database is improved. This last effect is particularly evidend for $\sigma_{R}>0.1$. For smaller values of $\sigma_{R}$, the gain obtained by increase the number of sampling points in the database from $3 \times 3$ to $5 \times 5$ is not particularly strong. The $3 \times 3$ and $5 \times 5$ databases will be considered for the following analysis and for the predictive simulations.

The domain $\Omega_{H F}$ for the zonal POD simulations is chosen by defining a threshold on the reconstruction error $\left(\sigma_{R}=15 \% U_{\infty}\right)$ and by choosing a rectangular box sufficiently large to contains all the points characterised by an error larger than this threshold, for both the $3 \times 3$ and $5 \times 5$ databases:

$$
\Omega_{H F}=\left\{\mathbf{x}=\{x, y\}^{T}:-1<x<3,-0.5<y<0.5\right\} \supset\left\{\mathbf{x} \in \Omega: e(\mathbf{x})>0.15 U_{\infty}\right\} .
$$

In the following study the extension of $\Omega_{H F}$ will be kept constant while different databases will be used to build the POD basis required by the predictive zonal simulations: this makes it possible to verify the convergence of the zonal approach when the database is enriched.

The mesh resolution in the predictive zonal simulations is equal to the mesh resolution of the highfidelity simulations (100 points on the chord of the airfoil) and so there are $400 \times 100$ mesh points in $\Omega_{H F}$. The high-fidelity solver used for these simulations requires the imposition of boundary conditions for the variable $\mathbf{u}^{*}$ and the pressure at the end of the time step. For this reason, the POD velocity modes are built from snapshots of the variable $\mathbf{u}^{*}$ and also the coupling projection (Eq. 17) is performed with reference to this variable. Dirichlet boundary conditions are used in the zonal POD simulation for both velocity and pressure.

The measured computational time of the zonal POD simulation is approximately 60 times smaller then a corresponding high-fidelity simulation performed on $\Omega$ in the considered setup. This large speed-up is related to the fact that uniform cartesian meshes are adopted in this test case. The use of adaptive non-uniform meshes would reduce the cost of an high-fidelity simulation in $\Omega$ and the benefits of the zonal POD approach. However, the need to accurately describe the translation of the gust from its initial position to the airfoil would require a significant number of degrees of freedom in the far field even in the presence of adaptive non-uniform meshes [31]. For this reason, the zonal POD approach can be useful in this kind of problems even when using non-uniform meshes.

The overlapping region is chosen according to the procedure reported in Section 4.3 and applied to the velocity magnitude error indicator. The overlapping region obtained by this study is used to perform the projection for both velocity and pressure in the zonal POD simulation. In particular, ten values of the threshold $\sigma_{O}$ are tested by uniformly increasing $\sigma_{O}$ from $1 \%$ to $10 \%$ of the far field velocity magnitude. When the $3 \times 3$ and $5 \times 5$ databases are considered, there are 9 and 25 sampling points, respectively. Therefore 90 and 250 zonal POD simulations are performed offline during this preliminary study. The 
Table 2: Threshold $\sigma_{O}$ and percentage number of points in the overlapping region $\left(N_{\Omega_{O}} / N_{\Omega_{H F}}\right)$

\begin{tabular}{|c|c|c|c|c|c|c|c|c|c|c|}
\hline$\sigma_{O} / U_{\infty}$ & $1 \%$ & $2 \%$ & $3 \%$ & $4 \%$ & $5 \%$ & $6 \%$ & $7 \%$ & $8 \%$ & $9 \%$ & $10 \%$ \\
\hline$N_{\Omega_{O}} / N_{\Omega_{H F}}$ & $1.1 \%$ & $4.1 \%$ & $6.9 \%$ & $12 \%$ & $18 \%$ & $29 \%$ & $39 \%$ & $47 \%$ & $55 \%$ & $63 \%$ \\
\hline
\end{tabular}

computational cost related to this analysis is affordable because a zonal POD simulation is 60 times faster than a high-fidelity simulation in the considered setup and so the cost of this analysis is comparable to the cost of a few high-fidelity simulations.

The percentage of mesh points in $\Omega_{O}$ with respect to the total number of mesh points in $\Omega_{H F}$ is reported in Table 2 as a function of the threshold $\sigma_{O}$ for the analysis performed on the $3 \times 3$ database. A similar behaviour is obtained for the $5 \times 5$ database.

The results of the study of the choice of $\Omega_{O}$ for the database $3 x 3$ are reported in Figures 8 and 9 which refer to different criteria for the evaluation of the quality of the zonal POD simulations. In Figure 8 the error on the predicted maximum lift coefficient $\left(C_{l}^{\max }\right)$ is reported as a function of $\sigma_{O}$. This error represents a key parameter because the maximum lift coefficient is a fundamental quantity required during the design of the structure. The plot shows that, in the considered example, the best choice for the overlapping region can be obtained by setting the threshold on the reconstruction error between $\sigma_{O}=6 \%$ and $\sigma_{O}=7 \%$ of $U_{\infty}$. This optimum choice corresponds to include between $29 \%$ and $39 \%$ of the points of $\Omega_{H F}$ into $\Omega_{O}$, according to Table 2 .

An alternative criterium can be defined by computing the L2-norm of the lift coefficient in the simulated time interval. The plot of this error as a function of $\sigma_{O}$ is reported in Figure 9. In this case the error reduces very slowly for $\sigma_{0} \geq 7 \%$ of $U_{\infty}$.

Both the considered criteria show that, in the considered test case, the prediction error grows when the threshold is chosen too small. This could be explained by observing that the choice of a small threshold will leave only a few points in the overlapping regions. In particular, the selected points are characterised by the lowest reconstruction error: usually this corresponds to choose the points in which all the snapshots are almost identical and the modes are almost zero. In this configuration, the least square minimisation used for the coupling (Eqs. 17 and 18) can lead to inaccurate results.

When the threshold is increased the two considered criteria show a different behaviour. The prediction error on the maximum lift coefficient has a mimimum and then tends to increase. The effect could be explained by the fact that when the threshold is too large then the overlapping region includes points in which the POD basis is not able to properly predict the new high-fidelity solution: the attempt to minimise the distance between the two solutions in these regions could deteriorate the reconstruction in the other regions.

In contrast, the L2-norm error on the lift coefficient evaluated during the simulated time interval shows a monotone decreasing behaviour when $\sigma_{O}$ is increased for almost all the configurations. This simple comparison shows that the choice of the overlapping region should be always related to the chosen goal function.

In the considered example all the configurations in the database give the minimum error for almost the same choice of the parameter $\sigma_{O}$. However, it is possible to get a more general behaviour in which different optimum values are obtained when exploring the space of parameters. In this case, a possible approach is to set $\sigma_{O}$ in a predictive zonal simulation equal to the optimal value obtained on the closest database sampling point during the offline analysis. This last general strategy will be adopted in the predictive simulations reported in the following.

\subsection{Predictive zonal POD simulations}

The zonal POD setup obtained by the analysis of the database is now used to perform some predictive simulations in order to test the ability of the method to predict the behaviour of the system for configurations which are not present in the database. 
First of all, a check is performed in order to quantify the contribution given by the POD modes to the definition of the boundary conditions in the zonal simulation. The results of this test are reported in Figure 10. The plot shows the evolution of the lift coefficient for a reference high-fidelity simulation performed on the large domain $\Omega$ and some predictive simulations performed on the small domain $\Omega_{H F}$ with different approaches.

A first prediction is done by using the high-fidelity solver on the small domain $\Omega_{H F}$ and by setting the same kind of boundary conditions used for the high-fidelity simulation in $\Omega$. Since the boundaries of the domain are now very close to the body, the flow field is strongly affected by the proximity of the boundary and so the solution is completely altered. Even the steady lift coefficient is very far from the reference value.

A better prediction can be performed by using the boundary conditions given by Eq. 12 with $N=0$ POD modes. This means that the boundary condition will be equal to the undisturbed steady field (computed on $\Omega$ ) plus the forcing terms which describe the advection of the vortex. This approach improves significantly the quality of the results and can be considered as an improved boundary condition with a correction term defined a priori. However, this approach misses the interaction between the vortex and the body.

Finally, a zonal POD simulation (with $N=45$ POD modes built from the $3 \times 3$ database) is performed in order to show how the introduction of the POD modes improves the boundary conditions.

A second test is performed to verify that effects of the choice of the overlapping region on the quality of a predictive zonal simulation. Figure 11 shows the history of the lift coefficient for a test configuration not-included in the database. The plot shows a comparison between the predictions obtained by the zonal POD approach for three different choices of the overlapping region $\left(\sigma_{O}=5 \%, 7 \%\right.$ and $9 \%$ of $\left.U_{\infty}\right)$ and the reference values obtained by a high-fidelity simulation. The POD basis is obtained from the $3 \times 3$ database. The effects of the choice of the overlapping region are clearly visible in the Figure 11 . The results obtained by setting the tolerance $\sigma_{O}=7 \%$ of $U_{\infty}$ show a good prediction of the maximum lift coefficient. However, larger errors can be seen after the first oscillation (see for example at the dimensionless time $=4)$. In contrast, the result obtained by setting $\sigma_{O}=9 \%$ of $U_{\infty}$ show a smaller averaged distance from the reference solution but they are characterised by a larger error on the maximum lift coefficient. The plot shows also the results obtained by setting $\sigma_{O}=5 \%$ which are characterised by larger errors with respect to both the previous optimal choices.

Finally, the ability of the zonal approach to predict new configurations is investigated in 25 test configurations generated by using the pseudo-random Sobol sequence. Their position in the parameter space is reported in Figure 4. In each zonal simulation the parameter $\sigma_{O}$ is chosen equal to the optimal value related to the closest database configuration in the space of the parameters, following the results of the offline analysis reported in Section 5.3. In order to evaluate the error of the predictions, 25 highfidelity simulations are performed on $\Omega$ and used as a reference. The results are reported in Figures 12 and 13, for the percentage error on $C_{l}^{\max }$ and the L2-norm error on the $C_{l}$, respectively.Both figures show in the top that the prediction error becomes very large when the POD reconstruction is not included in the definition of the boundary conditions (which means setting $N=0$ in Eq. 12). However, when 45 modes of the POD basis obtained from the $3 \times 3$ database (middle) and $5 \times 5$ database (bottom) are included, the error is significantly reduced. 

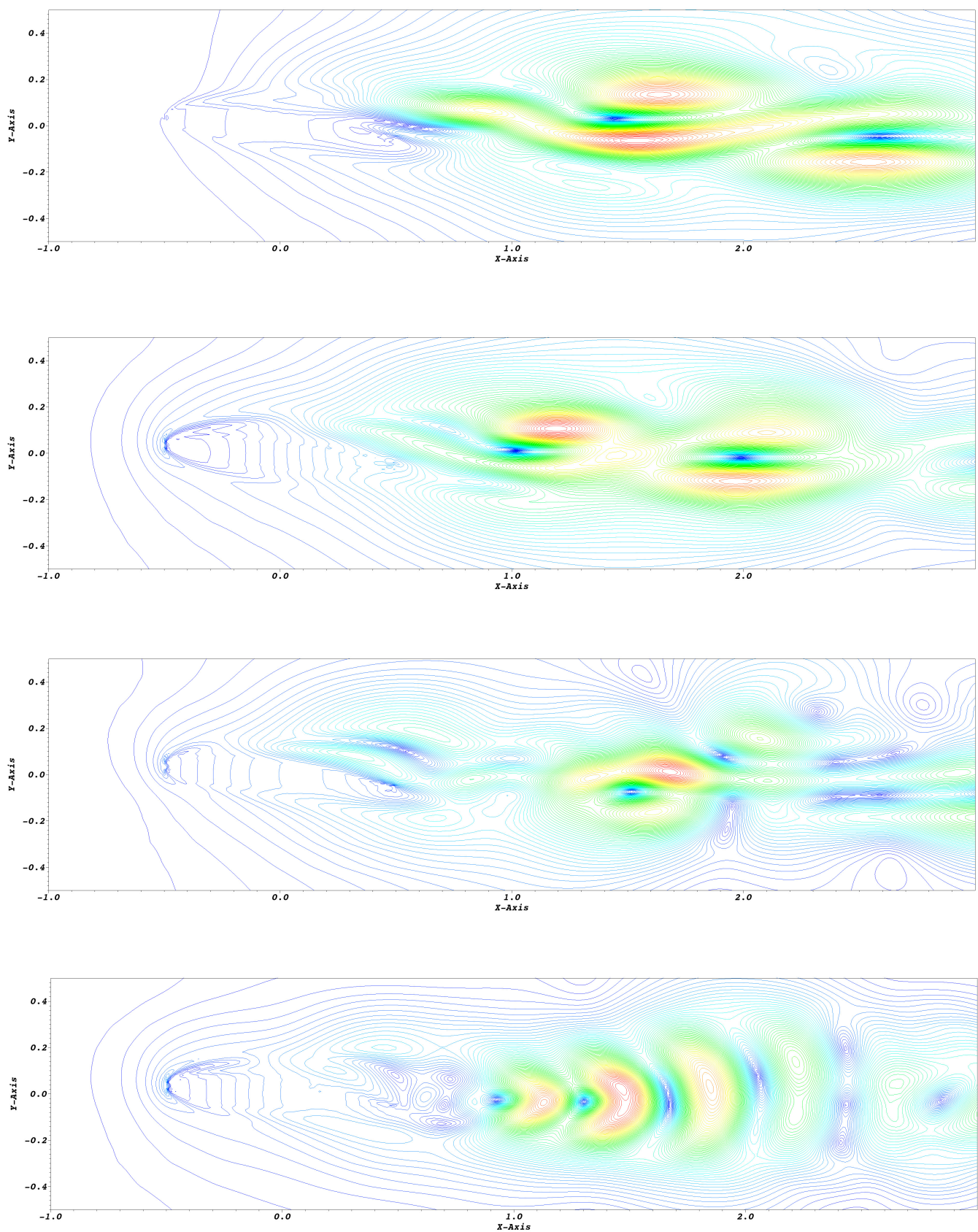

Figure 3: First four POD modes for the velocity magnitude field (energy decreasing from top to bottom). 


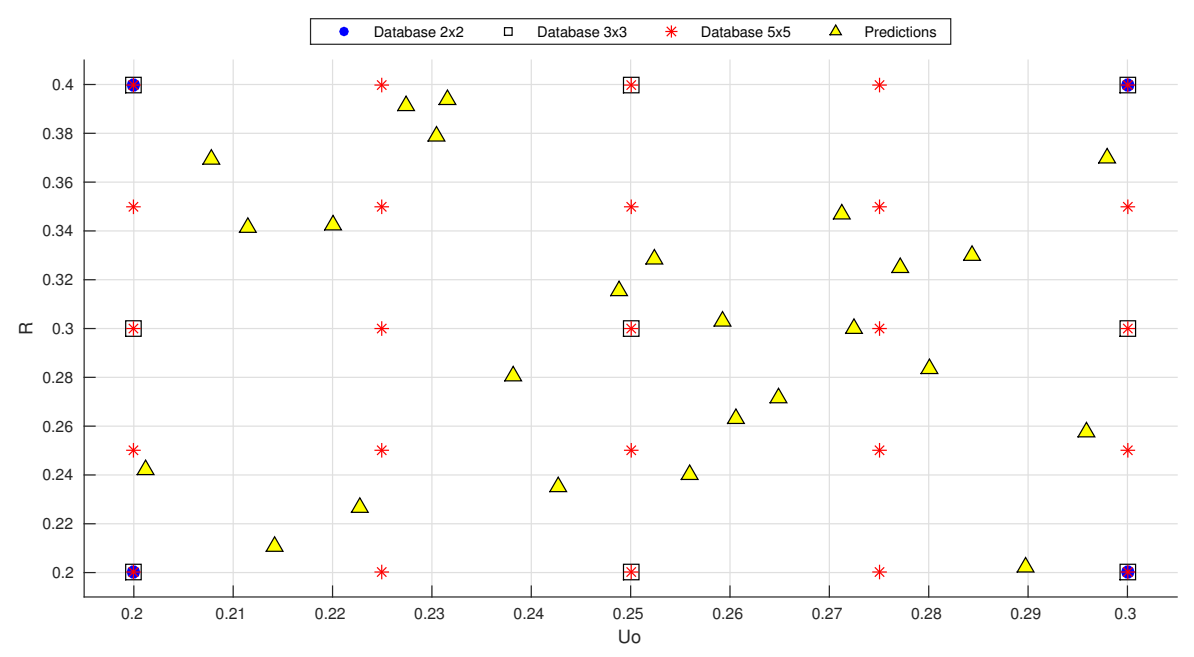

Figure 4: Gust parameters for the configurations used in the databases and in the predictive simulations

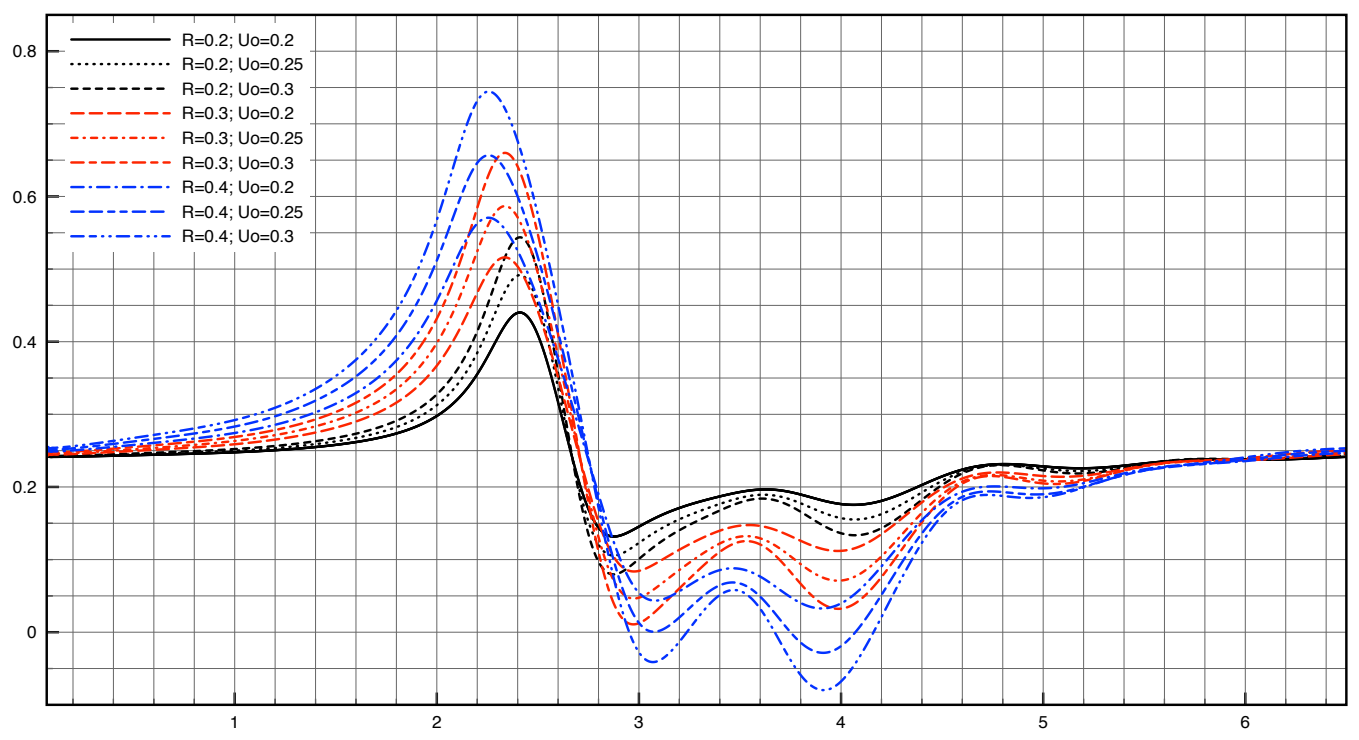

Figure 5: Lift coefficient history (High-fidelity simulations included in the $3 \times 3$ database). 

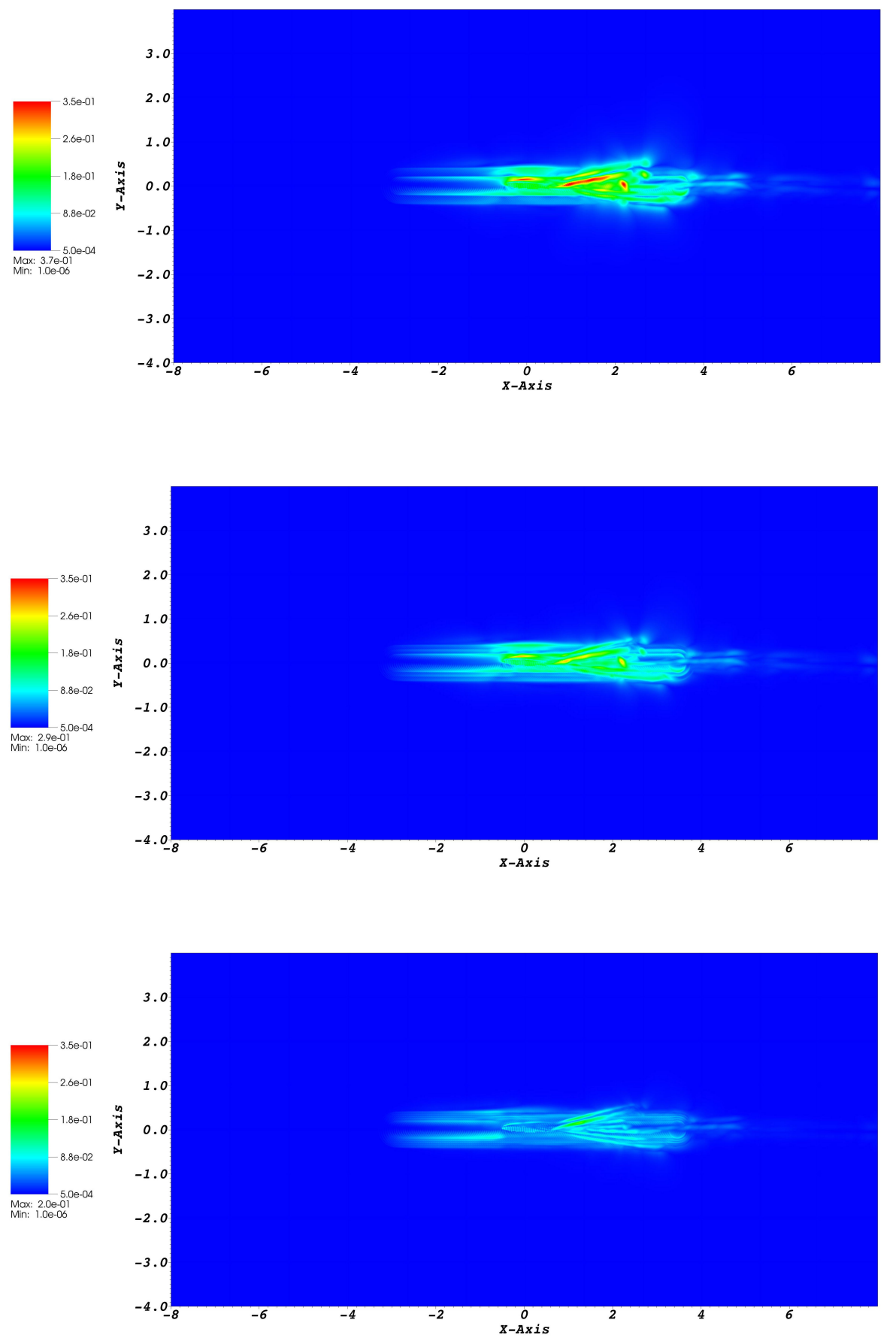

Figure 6: Prediction error indicator on the velocity magnitude (normalised w.r.t. $U_{\infty}$ ) for $2 \times 2$ (top), $3 \times 3$ (middle) and $5 \times 5$ (bottom) databases 


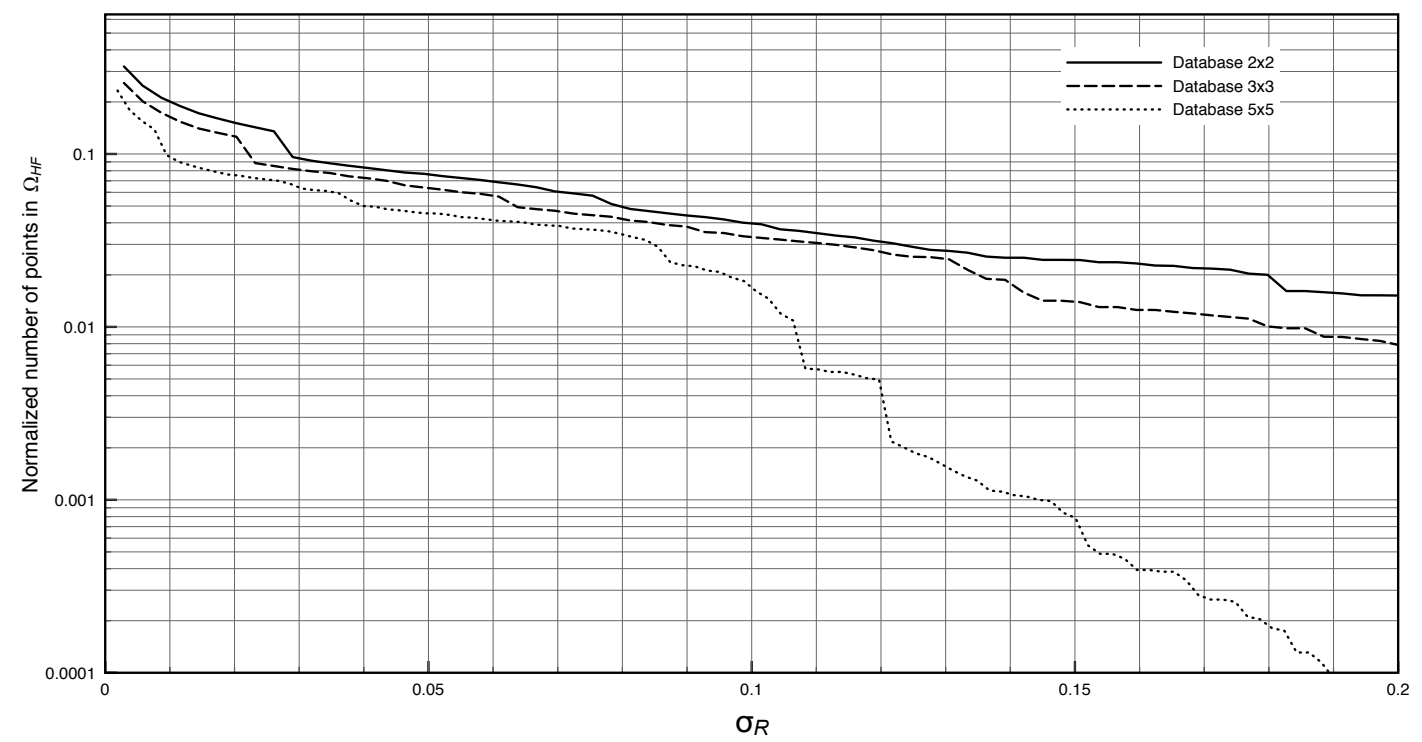

Figure 7: Number of mesh points in $\Omega_{H F}$ (normalised w.r.t. the number of mesh points in $\Omega$ ) as a function of $\sigma_{R}$ for different databases

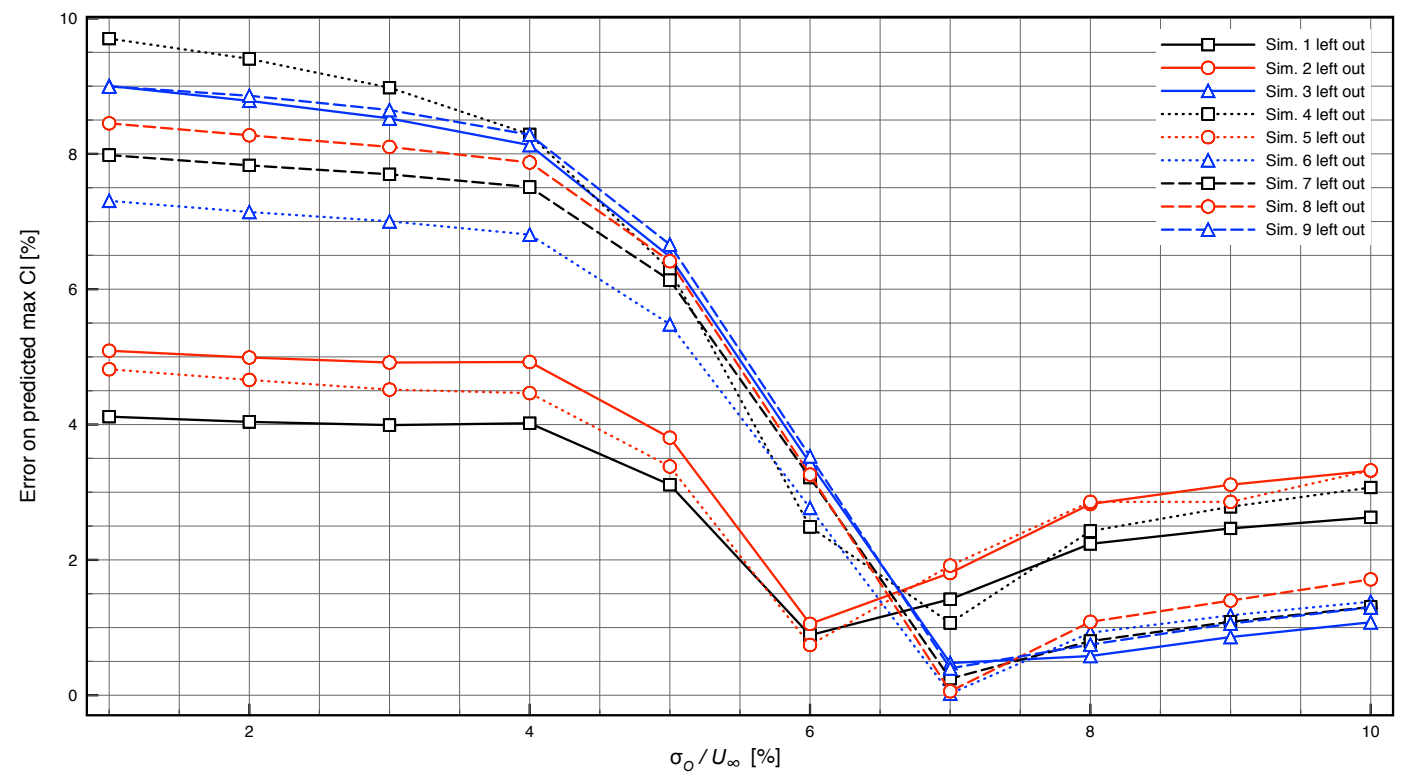

Figure 8: Error on the predicted max $C_{l}$ as a function of the threshold $\sigma_{O}$ (database $3 \times 3$ ) 


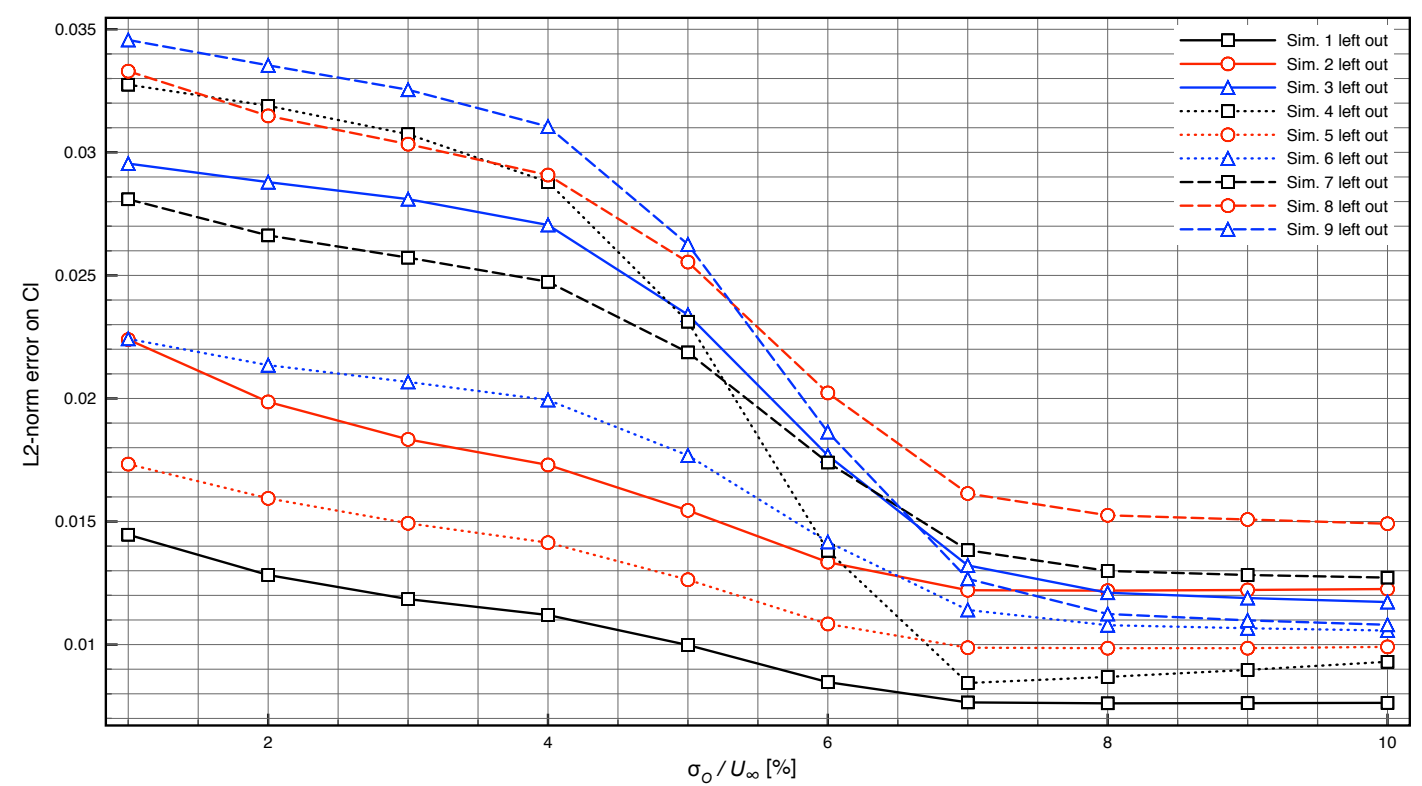

Figure 9: L2-norm error on the predicted $C_{l}$ as a function of the threshold $\sigma_{O}$ (database $3 \times 3$ )

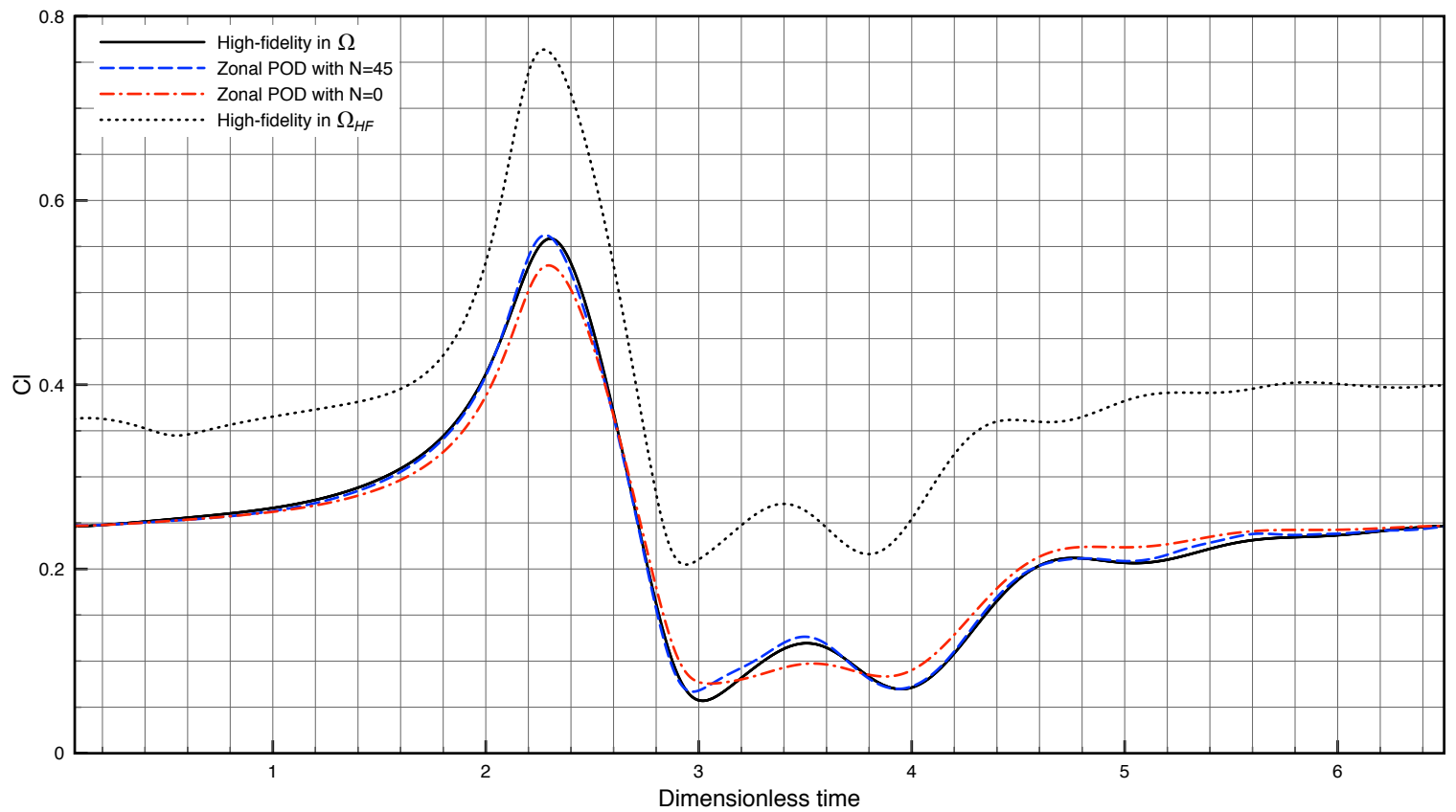

Figure 10: Evaluation of the contribution given by the POD modes to the boundary conditions of a predictive zonal simulation $\left(U_{0}=0.21, R=0.34\right)$ 


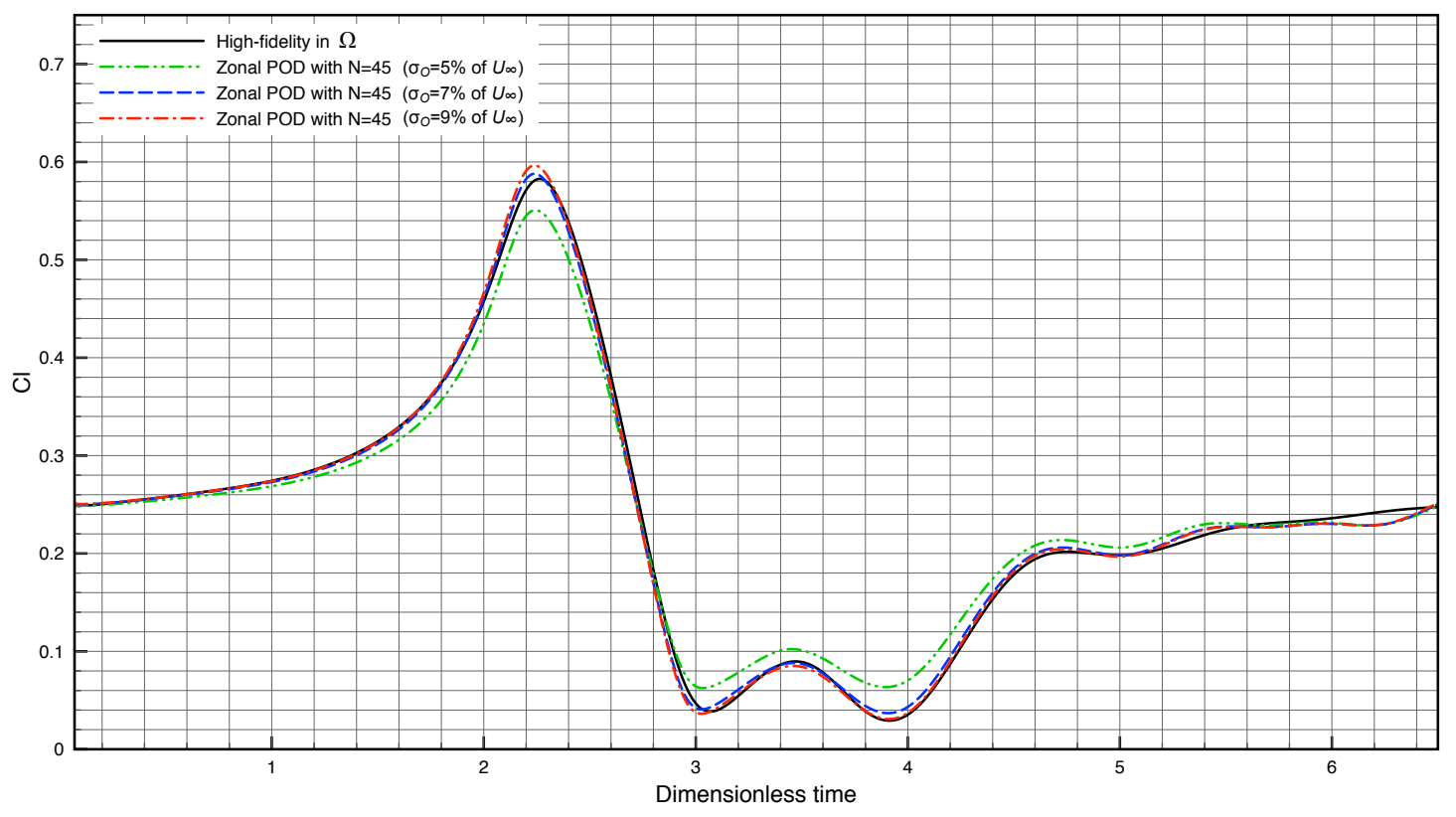

Figure 11: Comparison between predictive zonal POD simulations and high-fidelity results $\left(U_{0}=0.21\right.$, $R=0.39)$ 

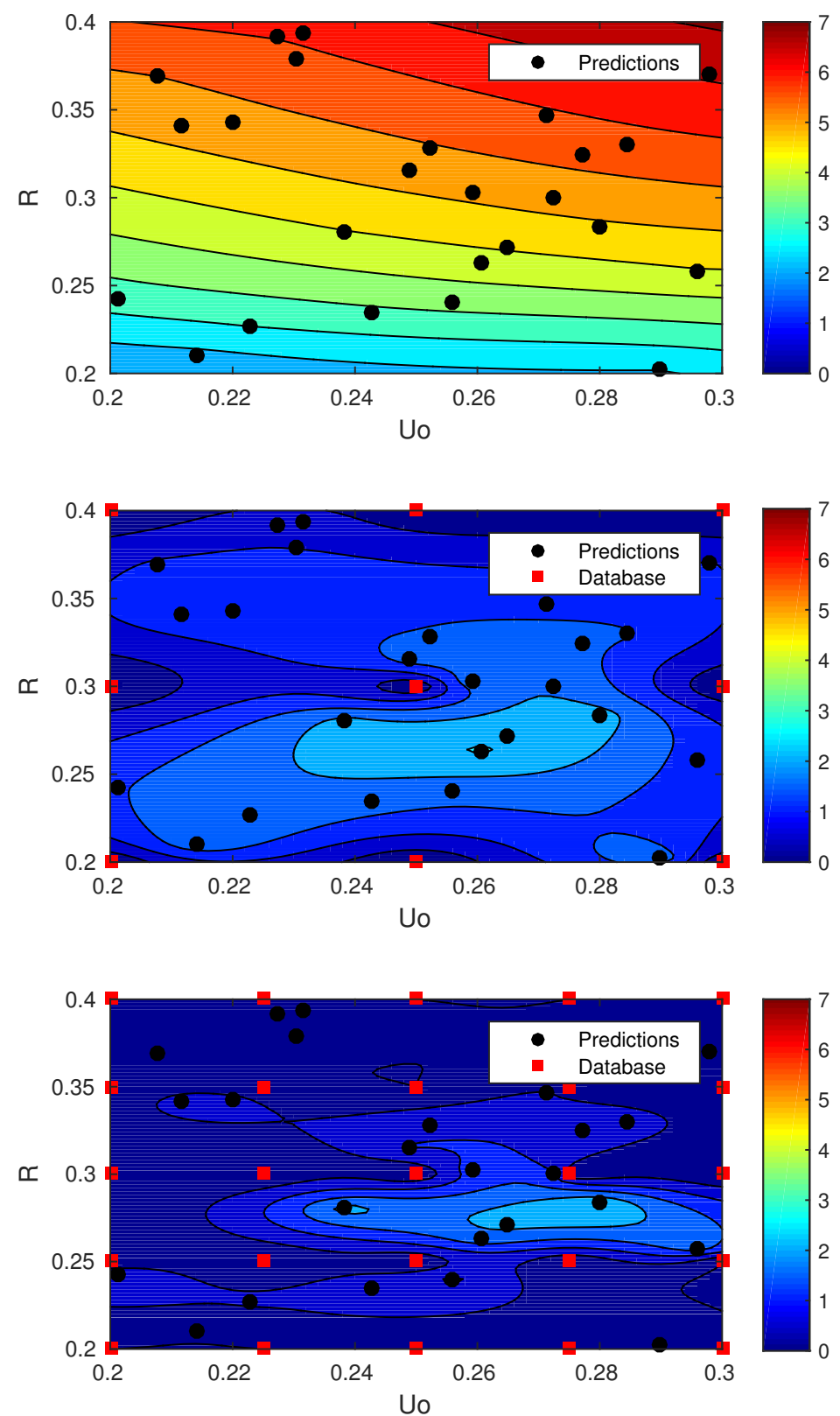

Figure 12: Percentage error on the $C_{l}^{\max }$ for predictive zonal simulation with $N=0$ (top), $N=45$ from database $3 \times 3$ (middle) and $N=45$ from database $5 \times 5$ (bottom) 

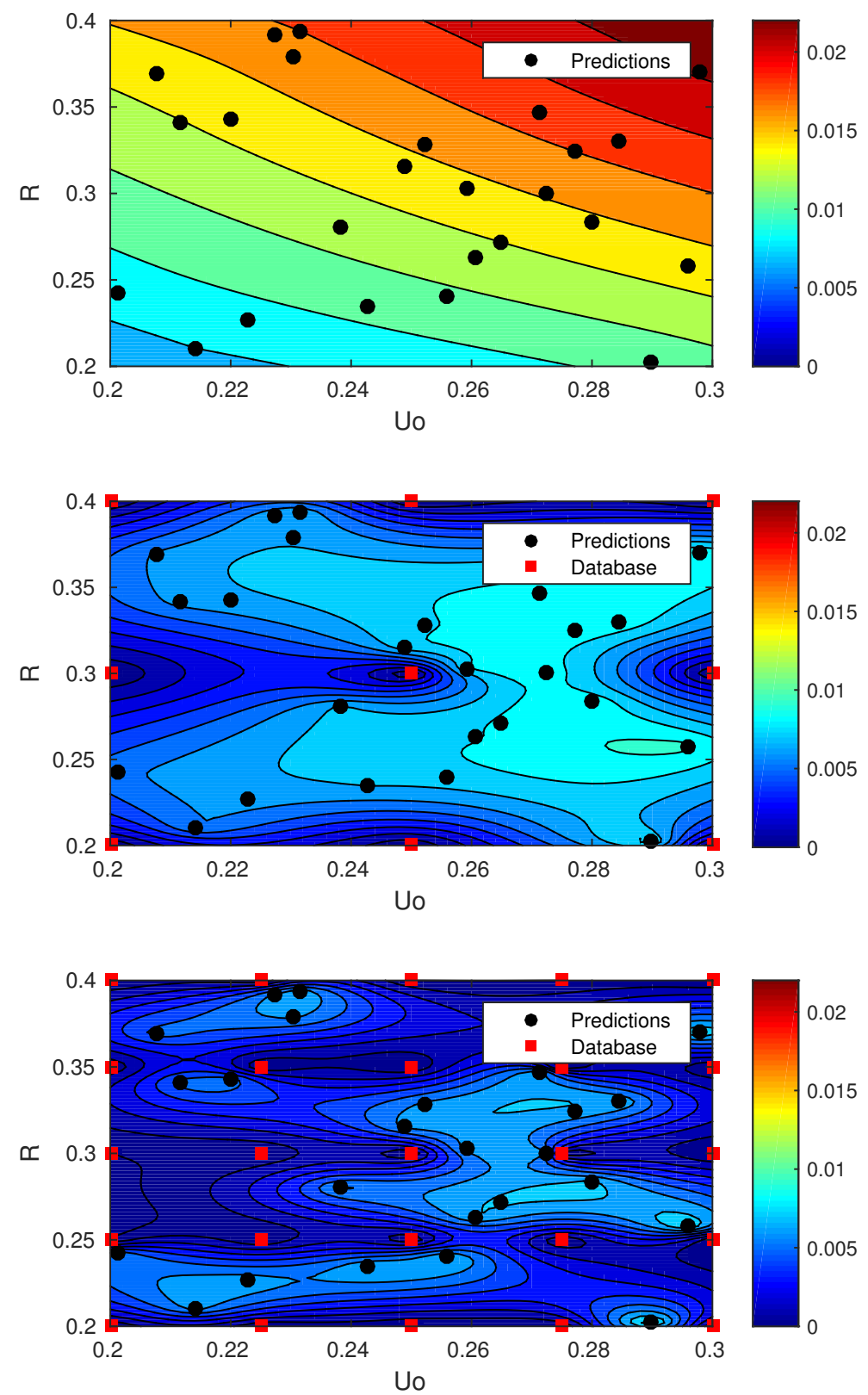

Figure 13: L2-norm error on the $C l$ for predictive zonal simulation with $N=0$ (top), $N=45$ from database $3 \times 3$ (middle) and $N=45$ from database $5 \times 5$ (bottom)

\section{A steady turbulent problem: aerodynamic shape optimiza- tion of a car section}

In this Section, the zonal POD approach is used to speed-up the simulations required by an aerodynamic shape optimization problem, i.e. the shape optimization of the front bumper of a $2 \mathrm{D}$ car section, aimed 


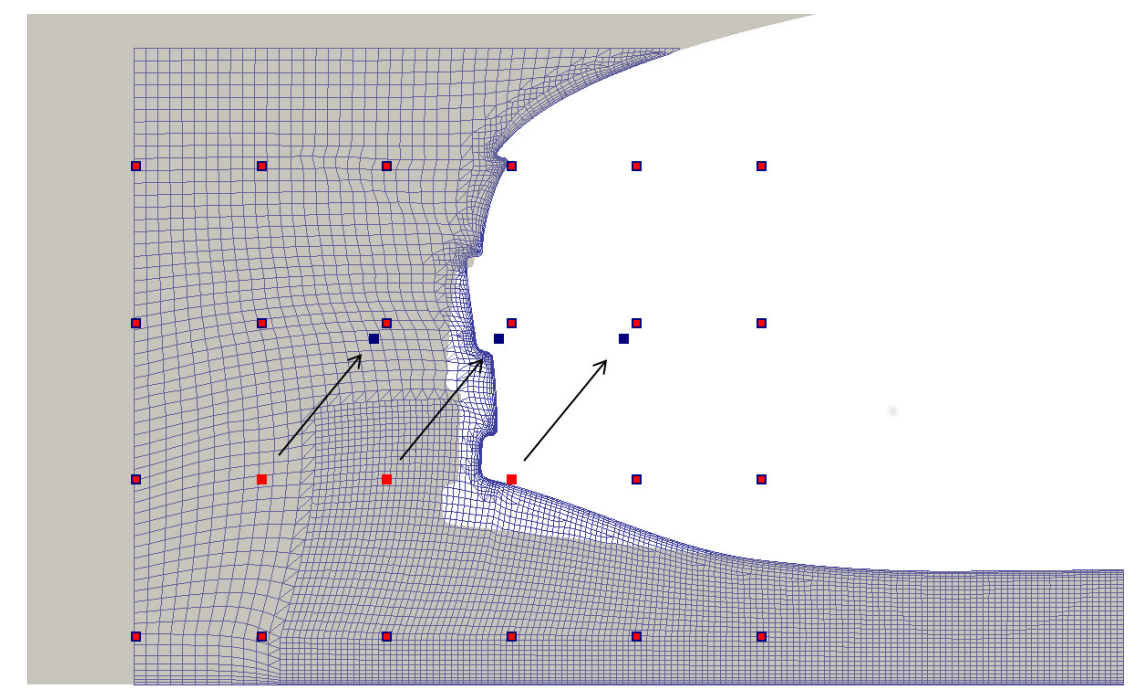

Figure 14: Mesh morphing: baseline configuration (red control points) and deformed configuration (blue control points).

at minimising the overall drag coefficient $\left(C_{d}\right)$.

Evolutionary algorithms typically require a large number of high-fidelity functional evaluations in order to find the global optimum. Therefore the computational cost of the optimization process is often prohibitive for large-scale aerodynamics problems. Here the zonal POD approach is used to provide low-cost function evaluations during the optimisation process.

A database of high-fidelity simulations is obtained by varying the bumper geometry and solving the RANS equations in the corresponding domain, using the method described in Section 2.2. All the simulations are performed at a Reynolds number equal to $R e=4.87 \cdot 10^{6}$, employing the Spalart-Allmaras turbulence model [51] and wall functions for near-wall treatment.

The computational domain $\Omega$ is discretized by a hex-dominant polyhedral mesh of 93388 cells, generated for the baseline configuration by means of snappyHexMesh, the mesh generation utility supplied with OpenFOAM. This mesh is then deformed through a Free-Form Deformation (FFD) technique [48] in order to obtain the meshes corresponding to other bumper configurations. For this application we use the PyGeM python library [1]. The parametrization is localized in the front area and the deformation is controlled by a lattice of $4 \times 6$ control points. Most of the points are fixed in order to assure a smooth transition between the deformed and the fixed region of the domain: only the three points highlighted in Figure 14 are allowed to move in the $x-y$ plane. The same displacement is applied to the three control points, resulting in only two design parameters for the optimization process ( $x_{c p}$ and $z_{c p}$ displacements).

The proposed methodology has been integrated into the OpenFOAM toolbox, in order to enable the analysis of complex geometries and different physical models.

\subsection{Analysis of the database: choice of the zonal setup}

The database is created considering different values of the parameters in the range $-0.18 \leq x_{c p} \leq 0.18$ and $-0.30 \leq z_{c p} \leq 0.30$. As in Section 5.3, the sampling is uniform and three different databases with $2 \times 2,3 \times 3$ and $5 \times 5$ points are considered (see Figure 15). Since steady RANS simulations are performed, only one snapshot is obtained for each sampling point $\left(N_{s}^{k}=1\right.$ and $N_{s}=N_{s p}$.

A separate POD basis is then computed for each physical quantity (i.e. $\mathbf{u}, p$ and Spalart-Allmaras variable $\tilde{\nu}$ ), after pre-processing the fields by choosing the forcing terms of Eq. 12 as the average fields among the snapshots. As a consequence, the maximum size of the POD basis will be equal to $N_{s}-1$. 


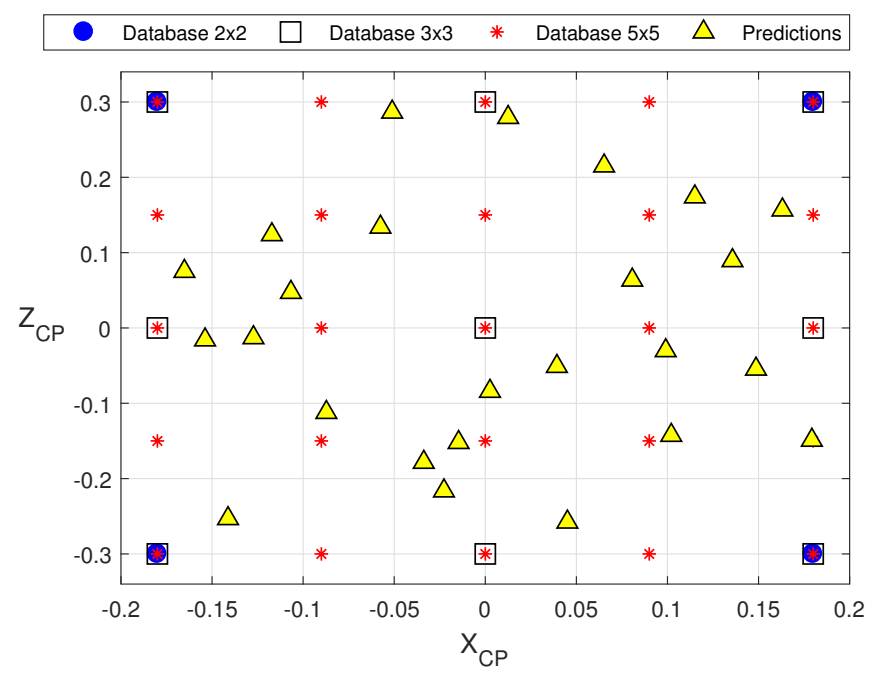

Figure 15: Control points displacements used in the databases and in the predictive simulations.

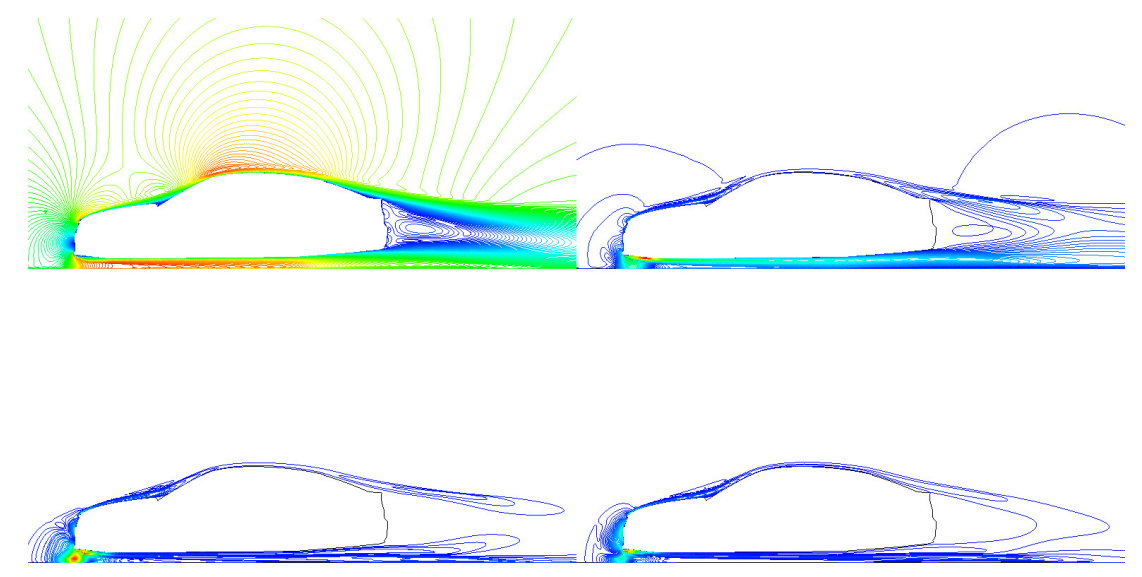

Figure 16: Magnitude of the mean velocity field (top right) and of the first three velocity POD modes (energy decreasing from top to bottom and left to right).

The velocity mean field and first three modes are shown in Figure 16.

Using the leave-one-out strategy described in Section 4.2, it is possible to compute the prediction error indicator map for all the physical quantities. The larger error values are localised close to the front bumper and in the turbulent wakes. Figure 17 shows the reduction of the maximum error on the velocity magnitude obtained by enriching the initial database. For this application, the initial database of 9 snapshots shows sufficiently small errors and therefore it will be used in the following analysis and during the optimization. The RIC analysis described in Section 3.1 showed that in this test case all the snaphots introduce relevant information and so we set the size of the POD basis equal to $N=N_{s}-1=8$.

$\Omega_{H F}$, i.e. the domain used for the zonal POD simulations, is a quasi-rectangular box containing the deformable part of the geometry and all the cells characterized by a prediction error $\sigma_{R}$ larger than a certain percentage of the far field velocity magnitude $U_{\infty}$. The mesh in $\Omega_{H F}$ is a subset of the original mesh in $\Omega$, and therefore it maintains the same resolution and topology. Figure 18 shows how the size of $\Omega_{H F}$ for a given $\sigma_{R}$ can be reduced by enriching the initial database of solutions. We choose to include in $\Omega_{H F}$ all the cells where $\sigma_{R}>0.01 U_{\infty}$ : such domain contains 9551 cells, resulting in a reduction of the problem size by a factor 10 . 

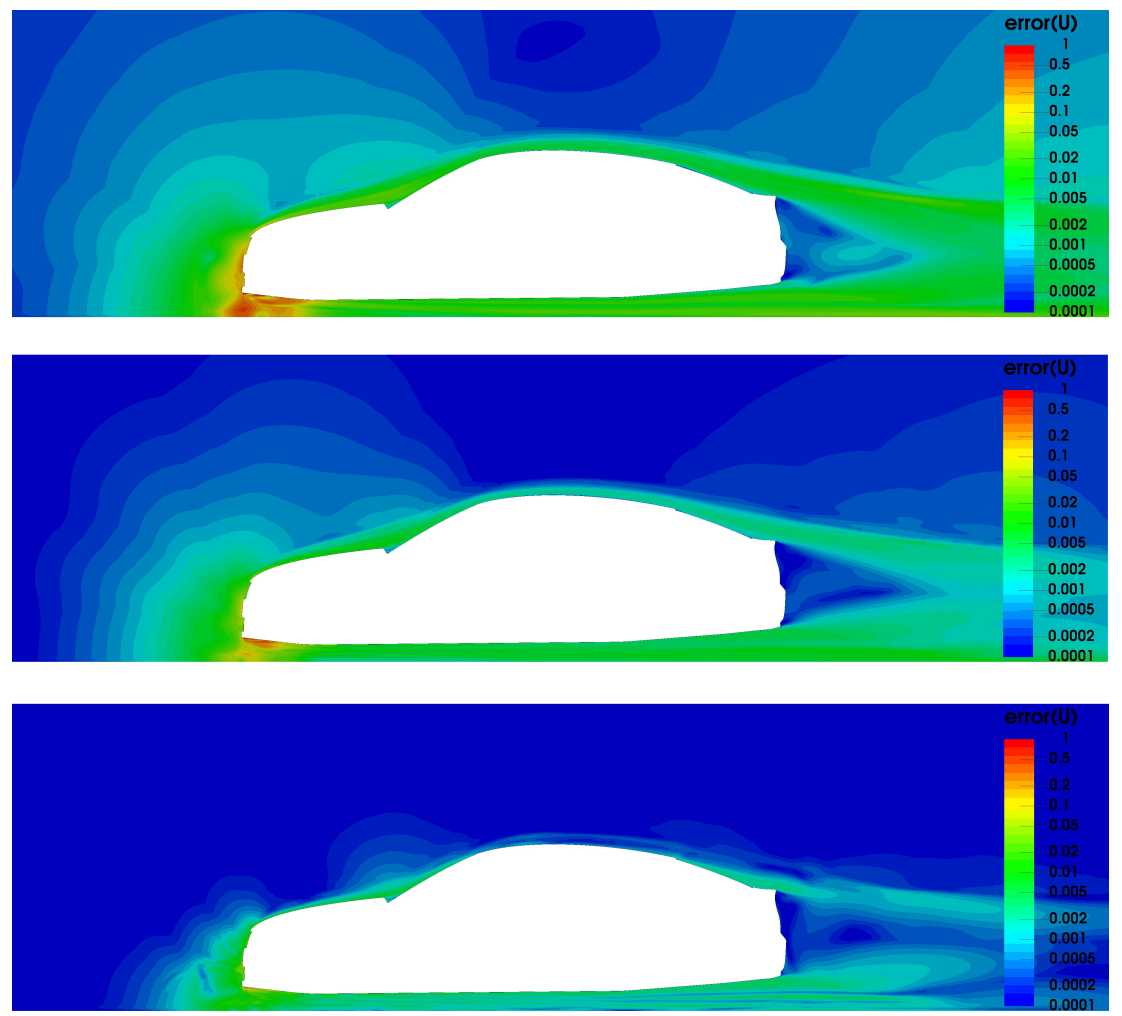

Figure 17: Prediction error indicator on the velocity magnitude (normalised w.r.t. $U_{\infty}$ ) for $2 \times 2$ (top), $3 \times 3$ (centre) and $5 \times 5$ (bottom) database.

The boundary conditions are updated at every solver iteration solving the minimisation problem described in Section 3.2. Dirichlet or Neumann conditions are imposed depending on the sign of the flux. For inflow boundaries, we use the POD modes to recover $\mathbf{u}$ and $\tilde{\nu}$ and the gradient of $p$, whereas for outflow boundaries we prescribe $p$ and the gradients of $\mathbf{u}$ and $\tilde{\nu}$ normal to the boundary. In order to obtain the global $C_{d}$ of the car, the converged solution is prolonged outside $\Omega_{H F}$, reconstructing the outer fields as a combination of the POD modes.

The relative importance of the two regions, i.e. $\Omega_{H F}$ and $\Omega \backslash \Omega_{H F}$, for the evaluation of the global aerodynamic coefficients is shown in Table 3 for a high-fidelity simulation not included in the database: the flow solution in $\Omega_{H F}$ accounts for approximately $31 \%$ of $C_{d}$ and $69 \%$ of $C_{l}$. Table 3 shows also the effectiveness of the zonal POD approach. In particular, we perform different zonal POD simulations, using $N_{s p}=9$ and $N_{s p}=4$ : as expected, the richer database guarantees a better approximation of the solution.

In order to evaluate the contribution given by the POD reconstruction to the definition of the BCs of the zonal simulation, we performed also a zonal simulation with $N=0$ POD modes (i.e. BCs derived only from the average field). The results reported in Table 3 show that the introduction of the POD modes reduces the percentage error on $C_{d}$ from $8.4 \%$ (when $N=0$ ) to $0.5 \%$ (when $N=8$ ). The same trend can be observed in Figure 19, where we report the distribution of the pressure coefficient $C_{p}$ on the vehicle underbody surface in both $\Omega_{H F}$ (solid lines) and $\Omega \backslash \Omega_{H F}$ (dotted lines).

The overlapping region is chosen by testing the configurations in the database (see Figure 15) for different values of the threshold $\sigma_{O}$, logarithmically spaced between $0.1 \%$ and $10 \%$ of $U_{\infty}$. Figure 20 shows the results obtained by the application of the leave-one-out method to the configurations belonging to the database $3 \times 3$. Here the quality of the choice of $\Omega_{O}$ is evaluated by checking the error of the reconstructed boundary conditions on $\partial \Omega_{H F}$. For this purpose, the error indicator $\epsilon_{O}$ is chosen as the L2-norm of the difference between the exact value of $\mathbf{u}$ evaluated on $\partial \Omega_{H F}$ and its POD reconstruction 


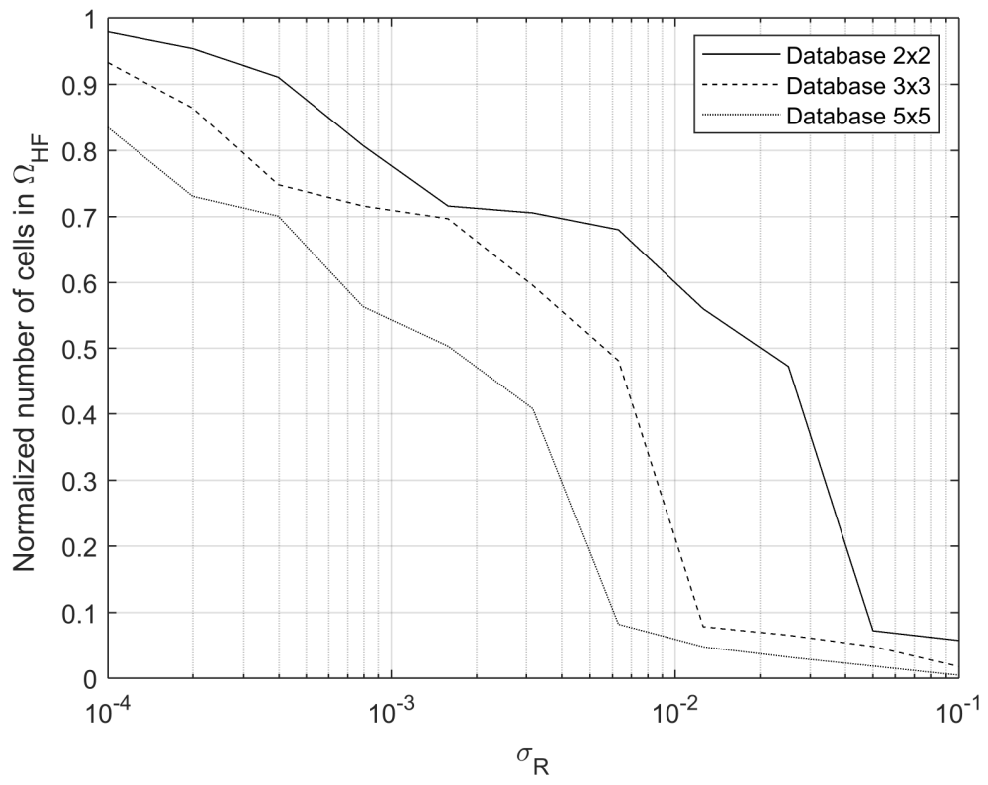

Figure 18: Number of cells in $\Omega_{H F}$ normalized with respect to the number of cells in $\Omega$ as a function of the prediction error threshold $\sigma_{R}$.

Table 3: Contribution of the flow solution in $\Omega_{H F}$ to the global aerodynamic coefficients for a predictive simulation $\left(x_{c p}=0.13568, z_{c p}=0.08935\right)$.

\begin{tabular}{|l|c|c|c|c|}
\hline \multirow{2}{*}{ Simulation type } & \multicolumn{2}{|c|}{$C_{d}$} & \multicolumn{2}{c|}{$C_{l}$} \\
\cline { 2 - 5 } & in $\Omega$ & in $\Omega_{H F}$ & in $\Omega$ & in $\Omega_{H F}$ \\
\hline High-Fidelity & 0.015075 & 0.004716 & -0.143092 & -0.099199 \\
\hline Zonal POD with $N_{s}=9$ and $N=0$ & 0.016346 & 0.006969 & $-0,120436$ & -0.096443 \\
\hline Zonal POD with $N_{s}=4$ and $N=3$ & 0.015271 & 0.004795 & -0.146132 & -0.100173 \\
\hline Zonal POD with $N_{s}=9$ and $N=8$ & 0.015153 & 0.004791 & -0.142726 & -0.098935 \\
\hline
\end{tabular}

calculated during the zonal simulation, normalised with the L2-norm of the exact velocity on $\partial \Omega_{H F}$.

In the considered sample, the approximation of the boundary conditions can be considered good for most of the thresholds: there is little variation with $\sigma_{O}$, meaning that it would be possible to take the whole $\Omega_{H F}$ as overlapping region. The best results for in-sample configurations are obtained for $\sigma_{O}=1.0 \%$ and $\sigma_{O}=1.7 \%$ of $U_{\infty}$. For the following optimization, we choose $\sigma_{O}=0.017 U_{\infty}$, with $\Omega_{O}$ including the $62.9 \%$ of the cells in $\Omega_{H F}$, as shown in Table 4 .

\subsection{Predictive zonal POD simulations}

The ability of the zonal approach to perform predictive simulations is investigated also for this application. As in Section 5.4, we test 25 configurations not included in the database and generated through a pseudo-random Sobol sequence (see Figure 15 for the distribution of the new points in the parameter space). The results are shown in Figure 21, where we report the percentage error on the overall $C_{d}$ with respect to the high-fidelity simulation. In particular, two different approaches are compared: in Figure 21(left) we set $N_{s}=9$ and $N=0$ (i.e. no POD reconstruction of the boundary conditions), whereas in Figure 21(right) $N_{s}=9$ and $N=8$. The zonal POD approach with $N=8$ behaves well on the parameter space, with an average error of $0.93 \%$ (maximum error $3.01 \%$ ) compared to the $7.00 \%$ (maximum error 14.83\%) obtained with $N=0$. The overlapping regions for the predictive simulations is fixed and chosen as described in Section 6.1. In Figure 22 we report the error of the reconstructed boundary conditions for different thresholds of $\sigma_{O}$ for two of the 25 predictive simulations: as expected, 


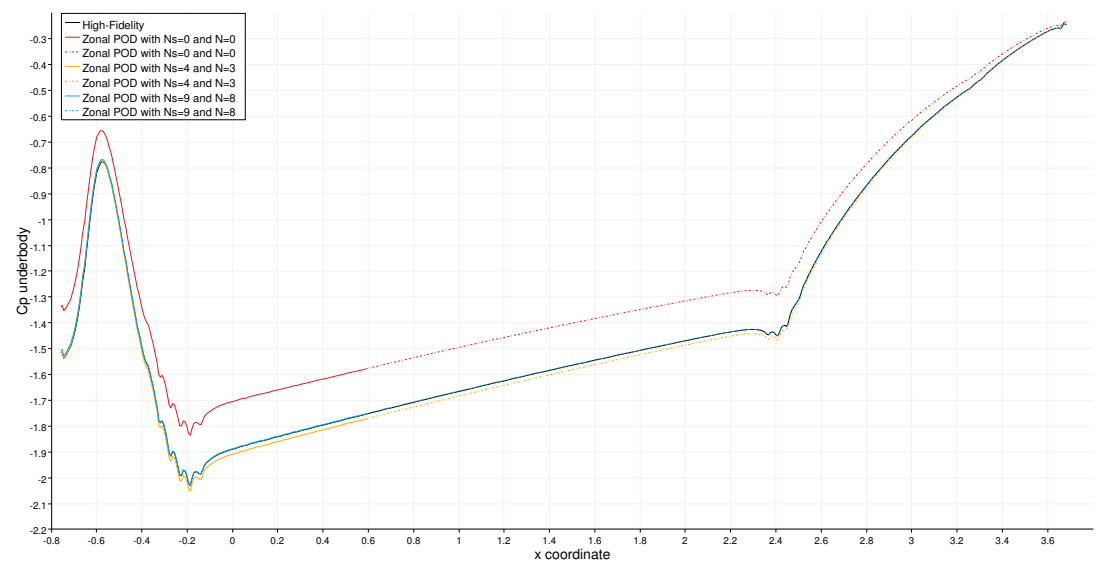

Figure 19: $C_{p}$ distribution at the underbody varying the POD reconstruction of the boundary conditions for a predictive simulation $\left(x_{c p}=0.13568, z_{c p}=0.08935\right)$ : contributions are represented by solid lines in $\Omega_{H F}$ and by dotted lines in $\Omega \backslash \Omega_{H F}$.

Table 4: Threshold $\sigma_{O}$ and percentual number of points in the overlapping region $\left(N_{O} / N_{H F}\right)$.

\begin{tabular}{|c|c|c|c|c|c|c|c|c|c|c|}
\hline$\sigma_{O} / U_{\infty}$ & $0.6 \%$ & $1.0 \%$ & $1.7 \%$ & $2.9 \%$ & $4.8 \%$ & $8.1 \%$ & $13.5 \%$ & $22.5 \%$ & $37.5 \%$ & $62.5 \%$ \\
\hline$N_{O} / N_{H F}$ & $40.7 \%$ & $51.0 \%$ & $62.9 \%$ & $73.0 \%$ & $88.7 \%$ & $95.5 \%$ & $98.2 \%$ & $99.3 \%$ & $99.9 \%$ & $100 \%$ \\
\hline
\end{tabular}

the trend is similar to the one observed in Figure 20.

\subsection{Optimisation framework}

The optimisation procedure is driven by an Efficient Global Optimization (EGO) algorithm [30], provided by the Dakota toolkit [2]. Starting from a sample of true simulations, a Gaussian Process approximation (kriging) of the objective function is build during each EGO iteration. Such true simulations are constituted by a limited number of high-fidelity simulations in $\Omega$ plus several zonal POD simulations. In particular, 9 high-fidelity simulations are performed in $\Omega$ using the simpleFoam solver for turbulent, incompressible flow, in order to generate the initial database of snapshots and build the corresponding POD basis. When the algorithm requires a new function evaluation to improve the kriging approximation of the response surface, a zonal POD is performed. The new point in the parameter space is chosen by maximising an Expected Improvement Function [2], providing balance between exploiting regions with good solutions and exploring regions where the prediction uncertainty is high. The zonal POD simulations are performed in a small subset of the original mesh, close to the deformed zone. Figure 23 shows a comparison between the extension of the computational domain used by the full-order simulations, $\Omega$, and the domain of the zonal POD simulations, $\Omega_{H F}$.

The overall drag coefficient of the car, i.e. the goal function of the optimization, is computed by summing the contribution obtained through the high-fidelity solver in $\Omega_{H F}$ and the one given by the POD reconstruction on $\Omega \backslash \Omega_{H F}$.

\subsection{Results}

The optimisation process is stopped when the Expected Improvement Function becomes smaller than a given threshold [2]. The optimum is found after 23 zonal POD functional evaluations. In terms of computational costs, the zonal approach allows to significantly reduce the total cost of the optimization process compared to a strategy based on high-fidelity simulations performed on $\Omega$. The computational time required by a high-fidelity simulation in $\Omega$ is approximately equal to $4000 \mathrm{~s}$, whereas a zonal POD simulation needs roughly $400 \mathrm{~s}$. As a result, if we consider the overall cost of the optimization, given by taking into account the 23 zonal simulations plus the 9 high-fidelity simulations in $\Omega$ required to build 


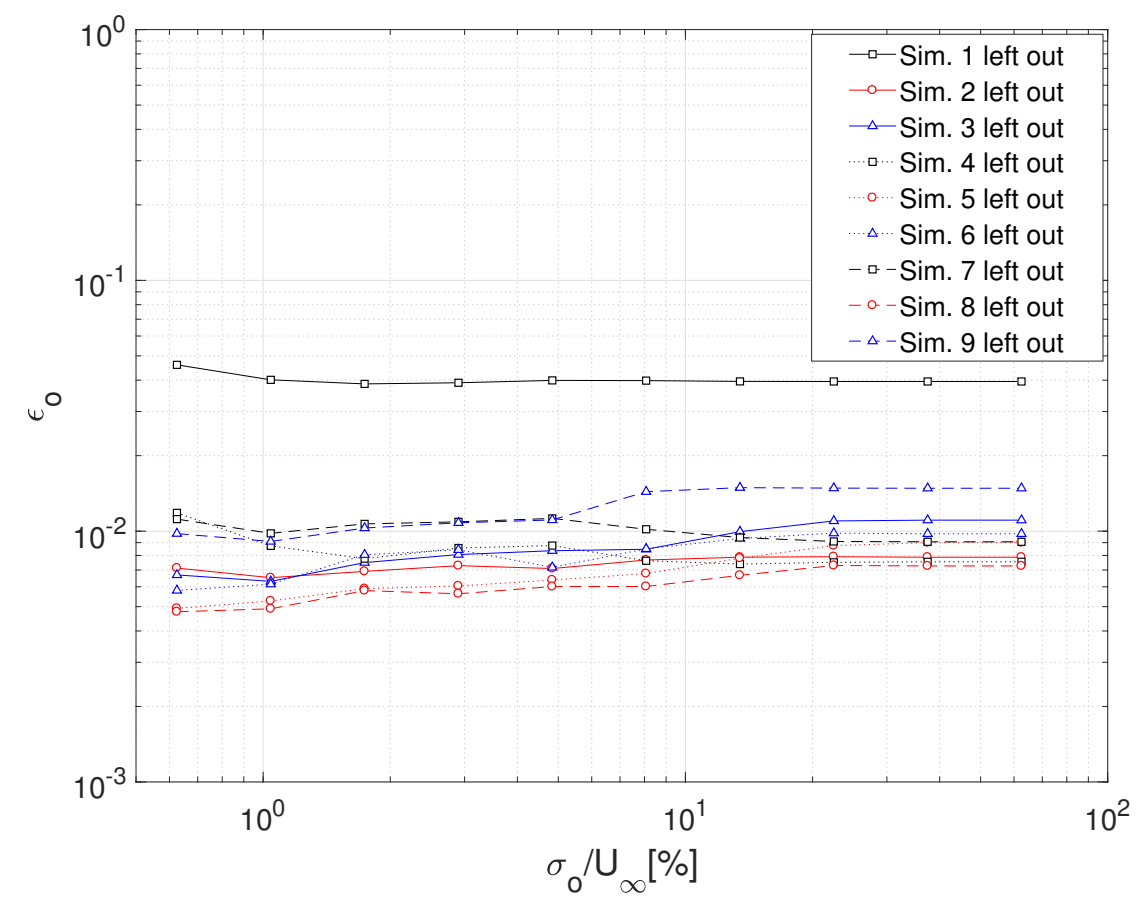

Figure 20: L2-norm error on the $\Omega_{H F}$ boundary conditions as a function of the threshold $\sigma_{O}$ (database $3 \times 3)$

the initial database, and compared it with the cost of an analogous optimization performed using only the full model, we save around the $65 \%$ of the computational time.

The response surface of the problem is reported in Figure 24 (left) where the drag coefficient is plotted as a function of the two design parameters. In Figure 24 (right), instead, it can be observed the variation of the relative error on $C_{d}$ between the reference high-fidelity solution and the zonal solution. With respect to the baseline configuration $\left(C_{d}=0.01527\right)$, the optimum configuration $\left(C_{d}=0.01456\right)$, evaluated with the zonal POD model, shows a reduction of the $4.6 \%$ with an error on the accuracy of the prediction smaller than $1.0 \%$. This trend is confirmed by the high-fidelity simulations performed a-posteriori.

\section{Conclusions}

A technique to perform zonal Galerkin-free POD simulations is presented. The approach requires an offline training for the definition of a POD basis obtained by some preliminary high-fidelity simulations. The POD basis is then exploited for predictive low cost zonal simulations. In particular, the computational domain in the zonal simulation is split between a low-fidelity and a high-fidelity region. The POD basis is used for the description of the external solution in the low-fidelity region while the high-fidelity solver is applied only in the regions where the flow field depends strongly on the choice of the design parameters. This makes the method suitable to perform predictive low cost simulations in the framework of an optimisation procedure.

In order to apply the zonal approach to the simulation of general flow fields it is necessary to clarify how to split the computational domain between the high-fidelity and the low-fidelity regions and how to couple the two solutions. Some possible techniques to address these problems during the offline training are proposed in this work.

A prediction error indicator based on a leave-one-out strategy is used to define an error map which allows to identify the regions where the POD basis cannot properly predict new configurations: these regions will be described by the high-fidelity solver during the zonal simulation. 

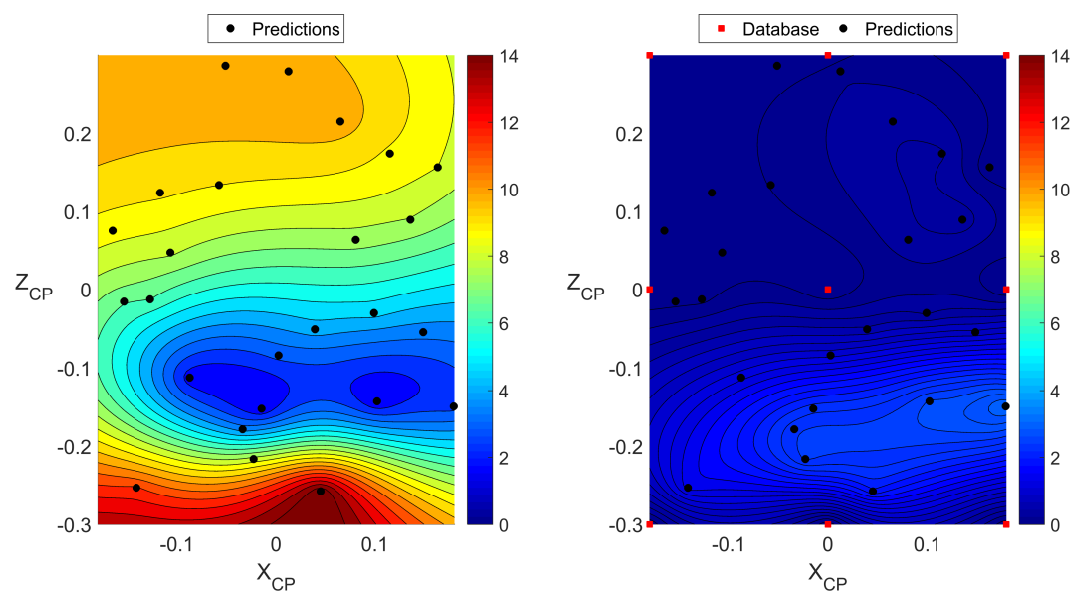

Figure 21: Percentage error on the overall $C_{d}$ predictive zonal simulationswith $N=0$ (left) and $N=8$ (right) using the $3 \times 3$ database

The leave-one-out strategy is also used to perform a preliminary study which allows to perform an optimal coupling between the high and low-fidelity solutions. In particular, the technique allows to identify an optimal overlapping zone which minimises the prediction error on a certain goal function.

The zonal approach is applied in this work to two different test cases: the simulation of the effects of a gust on an airfoil and the aerodynamic shape optimisation of a car section.

The first problem is studied by the introduction of some forcing terms in the ROM reconstruction which allows to generalise the zonal approach to problems with time-dependent far field boundary conditions. The obtained results show that the proposed approach can be used to perform predictive simulations.

The second test case shows a possible application of the zonal approach as a low cost predictive tool in the framework of shape optimisation procedures. In particular, the approach appears to be particularly convenient for problems in which only local perturbations of the geometry are considered. Similar benefits can be expected in multi-body configurations when the optimisation is focused on the shape of a single body.

An advantage of the zonal approach is that it can be easily implemented in an existing high-fidelity solver with respect to other acceleration techniques: it is just necessary to introduce a function for the projection of the solution onto the POD basis and the evaluation of the boundary conditions.

In conclusion, the zonal approach has been successfully applied to the unsteady laminar Navier-Stokes equations and to the steady RANS equations. The application of the method to the unsteady RANS equations should not introduce additional problems and should follow what is done for the unsteady laminar Navier-Stokes equations. However, the extension to the study of turbulent flows by means of Direct Numerical Simulations or Large Eddy Simulations is much more challenging because of the wide range of scales which appear in the solution and which require a very large POD basis.

\section{Acknowledgements}

This work was supported by the AEROGUST project which has been funded from the European Union's Horizon 2020 research and innovation programme under grant agreement No 636053. The partners in AEROGUST are: University of Bristol, INRIA, NLR, DLR, University of Cape Town, NUMECA, Optimad Engineering S.r.l., University of Liverpool, Airbus Defence and Space, Dassault Aviation, Piaggio Aerospace and Valeol.

Some experiments presented in this paper were carried out using the PLAFRIM experimental testbed, being developed under the Inria PlaFRIM development action with support from Bordeaux INP, LABRI 


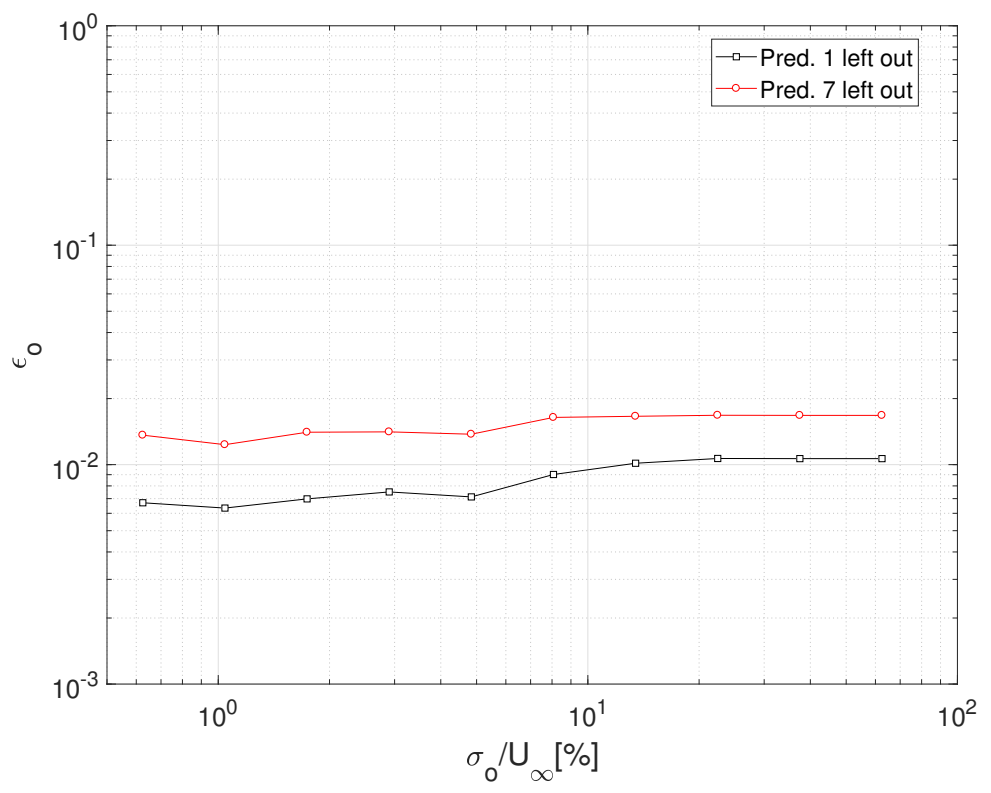

Figure 22: L2-norm error on the $\Omega_{H F}$ boundary conditions as a function of the threshold $\sigma_{O}$ for some predictive simulations

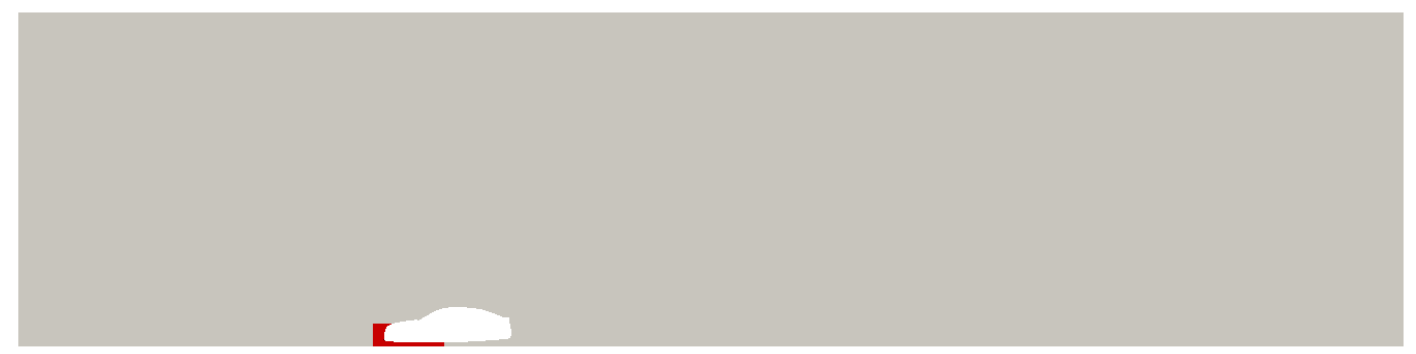

Figure 23: Full high-fidelity domain $\Omega$ (in grey) and zonal high-fidelity domain $\Omega_{H F}$ (in red).

and IMB and other entities: Conseil Régional d'Aquitaine, Université de Bordeaux and CNRS (and ANR in accordance to the programme Investissements d'Avenir (see https://www.plafrim.fr/).

Some simulations presented in this work were carried out using the MCIA computing platform (Mésocentre de Calcul Intensif Aquitain, see www.mcia.univ-bordeaux.fr).

\section{References}

\section{References}

[1] Pygem: Python Geometrical Morpher. https://github.com/mathLab/PyGeM/.

[2] B.M. Adams, L.E. Bauman, W.J. Bohnhoff, K.R. Dalbey, M.S. Ebeida, J.P. Eddy, M.S. Eldred, P.D. Hough, K.T. Hu, J.D. Jakeman, J.A. Stephens, L.P. Swiler, D.M. Vigil, and T.M. Wildey. Dakota, a multilevel parallel object-oriented framework for design optimization, parameter estimation, uncertainty quantification, and sensitivity analysis: Version 6.0 user's manual. Sandia National Laboratories, Tech. Rep. SAND2014-4633, 2014.

[3] David Amsallem, Matthew J Zahr, and Charbel Farhat. Nonlinear model order reduction based 

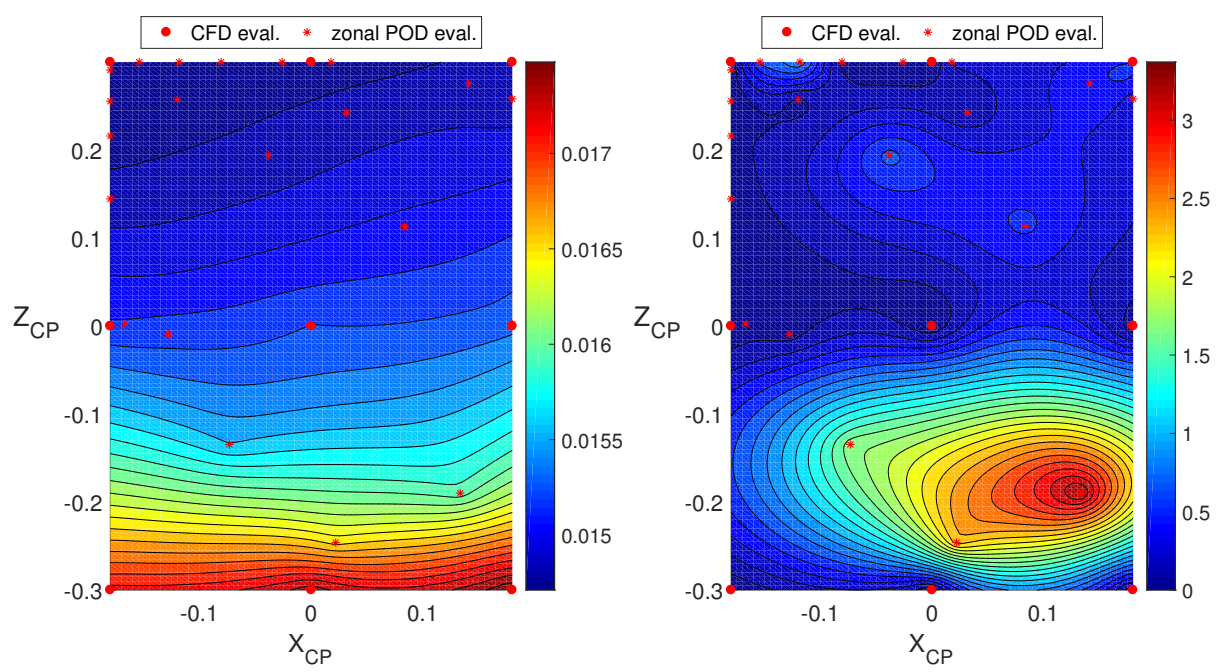

Figure 24: Drag coefficient as a function of the design parameters (left) and relative error of the zonal POD model with respect to the reference high-fidelity simulations (right).

on local reduced-order bases. International Journal for Numerical Methods in Engineering, 92(10): 891-916, 2012.

[4] Shervin Bagheri, Philipp Schlatter, Peter J Schmid, and Dan S Henningson. Global stability of a jet in crossflow. Journal of Fluid Mechanics, 624:33-44, 2009.

[5] Joan Baiges, Ramon Codina, and Sergio Idelsohn. Reduced-order subscales for pod models. Computer Methods in Applied Mechanics and Engineering, 291:173-196, 2015.

[6] Alexandre Barbagallo, Denis Sipp, and Peter J Schmid. Closed-loop control of an open cavity flow using reduced-order models. Journal of Fluid Mechanics, 641:1-50, 2009.

[7] Maxime Barrault, Yvon Maday, Ngoc Cuong Nguyen, and Anthony T Patera. An 'empirical interpolation' method: application to efficient reduced-basis discretization of partial differential equations. Comptes Rendus Mathematique, 339(9):667-672, 2004.

[8] Michel Bergmann, C-H Bruneau, and Angelo Iollo. Enablers for robust pod models. Journal of Computational Physics, 228(2):516-538, 2009.

[9] Michel Bergmann, Thierry Colin, Angelo Iollo, Damiano Lombardi, Olivier Saut, and Haysam Telib. Reduced order models at work in aeronautics and medicine. In Reduced Order Methods for Modeling and Computational Reduction, pages 305-332. Springer, 2014.

[10] Michel Bergmann, Jessica Hovnanian, and Angelo Iollo. An accurate cartesian method for incompressible flows with moving boundaries. Communications in Computational Physics, 15(05): 1266-1290, 2014.

[11] Jiri Blazek. Computational fluid dynamics: principles and applications. Butterworth-Heinemann, 2015.

[12] T Braconnier, M Ferrier, J-C Jouhaud, M Montagnac, and P Sagaut. Towards an adaptive pod/svd surrogate model for aeronautic design. Computers \& Fluids, 40(1):195-209, 2011.

[13] Marcelo Buffoni and Karen Willcox. Projection-based model reduction for reacting flows. In 40th Fluid Dynamics Conference and Exhibit, page 5008, 2010.

[14] Marcelo Buffoni, Haysam Telib, and Angelo Iollo. Iterative methods for model reduction by domain decomposition. Computers \&F Fluids, 38(6):1160-1167, 2009. 
[15] Alfonso Caiazzo, Traian Iliescu, Volker John, and Swetlana Schyschlowa. A numerical investigation of velocity-pressure reduced order models for incompressible flows. Journal of Computational Physics, 259:598-616, 2014.

[16] Kevin Carlberg and Charbel Farhat. A compact proper orthogonal decomposition basis for optimization-oriented reduced-order models. In 12th AIAA/ISSMO multidisciplinary analysis and optimization conference, page 5964, 2008.

[17] Kevin Carlberg, Charbel Bou-Mosleh, and Charbel Farhat. Efficient non-linear model reduction via a least-squares petrov-galerkin projection and compressive tensor approximations. International Journal for Numerical Methods in Engineering, 86(2):155-181, 2011.

[18] Saifon Chaturantabut and Danny C Sorensen. Nonlinear model reduction via discrete empirical interpolation. SIAM Journal on Scientific Computing, 32(5):2737-2764, 2010.

[19] Francisco Chinesta, Adrien Leygue, Felipe Bordeu, Jose Vicente Aguado, Elías Cueto, David González, Iciar Alfaro, Amine Ammar, and Antonio Huerta. Pgd-based computational vademecum for efficient design, optimization and control. Archives of Computational Methods in Engineering, 20(1):31-59, 2013.

[20] Alexandre Joel Chorin. Numerical solution of the navier-stokes equations. Mathematics of computation, 22(104):745-762, 1968.

[21] Tiberiu Coloşi, Mihail-Ioan Abrudean, Mihaela-Ligia Unguresan, and Vlad Muresan. Numerical simulation of distributed parameter processes. Springer, 2013.

[22] Federico Gallizio, Angelo Iollo, Bartosz Protas, and Luca Zannetti. On continuation of inviscid vortex patches. Physica D: Nonlinear Phenomena, 239(3):190-201, 2010.

[23] Seymour Geisser. Predictive inference, volume 55. CRC press, 1993.

[24] Reza Ghias, Rajat Mittal, and Haibo Dong. A sharp interface immersed boundary method for compressible viscous flows. Journal of Computational Physics, 225(1):528-553, 2007.

[25] Swetlana Giere, Traian Iliescu, Volker John, and David Wells. Supg reduced order models for convection-dominated convection-diffusion-reaction equations. Computer Methods in Applied Mechanics and Engineering, 289:454-474, 2015.

[26] Miloš Ilak and Clarence W Rowley. Modeling of transitional channel flow using balanced proper orthogonal decomposition. Physics of Fluids, 20(3):034103, 2008.

[27] H. Jasak. Error Analysis and Estimation for the Finite Volume Method with Applications to Fluid Flows. PhD thesis, Department of Mechanical Engineering - Imperial College, 1996.

[28] Hrvoje Jasak, Aleksandar Jemcov, and Zeljko Tukovic. Openfoam: A c++ library for complex physics simulations. In International workshop on coupled methods in numerical dynamics, volume 1000, pages 1-20. IUC Dubrovnik, Croatia, 2007.

[29] G Jin and M Braza. A nonreflecting outlet boundary condition for incompressible unsteady navierstokes calculations. Journal of computational physics, 107(2):239-253, 1993.

[30] Donald R Jones, Matthias Schonlau, and William J Welch. Efficient global optimization of expensive black-box functions. Journal of Global optimization, 13(4):455-492, 1998.

[31] Philip Kelleners and Ralf Heinrich. Simulation of interaction of aircraft with gust and resolved les-simulated atmospheric turbulence. In Advances in Simulation of Wing and Nacelle Stall, pages 203-221. Springer, 2016.

[32] Dilek Funda Kurtulus. On the unsteady behavior of the flow around naca 0012 airfoil with steady external conditions at re=1000. International Journal of Micro Air Vehicles, 7(3):301-326, 2015. 
[33] R. Mittal, H. Dong, M. Bozkurttas, F.M. Najjar, A. Vargas, and A. von Loebbecke. A versatile sharp interface immersed boundary method for incompressible flows with complex boundaries. Journal of Computational Physics, 227(10):4825 - 4852, 2008. ISSN 0021-9991.

[34] Bernd R Noack and Helmut Eckelmann. A global stability analysis of the steady and periodic cylinder wake. Journal of Fluid Mechanics, 270:297-330, 1994.

[35] Bernd R Noack and Helmut Eckelmann. A low-dimensional galerkin method for the threedimensional flow around a circular cylinder. Physics of Fluids, 6(1):124-143, 1994.

[36] Bernd R Noack, Paul Papas, and Peter A Monkewitz. The need for a pressure-term representation in empirical galerkin models of incompressible shear flows. Journal of Fluid Mechanics, 523:339-365, 2005.

[37] Bernd R Noack, Marek Morzynski, and Gilead Tadmor. Reduced-order modelling for flow control, volume 528. Springer Science \& Business Media, 2011.

[38] Jan Östh, Bernd Noack, Sinisa Krajnovic, Diogo Barros, and Jacquees Borée. On the need for a nonlinear subscale turbulence term in pod models as exemplified for a high-reynolds-number flow over an ahmed body. Journal of Fluid Mechanics, 747:518-544, 2014.

[39] S. V. Patankar. Numerical heat transfer and fluid flow. CRC press, 1980.

[40] S Perotto, A Reali, P Rusconi, and A Veneziani. Higamod: A hierarchical isogeometric approach for model reduction in curved pipes. Computers \& Fluids, 142:21-29, 2017.

[41] T J\&amp Poinsot and SK Lelef. Boundary conditions for direct simulations of compressible viscous flows. Journal of computational physics, 101(1):104-129, 1992.

[42] Alfio Quarteroni. Modeling the heart and the circulatory system, volume 14. Springer, 2015.

[43] Alfio Quarteroni and Gianluigi Rozza. Reduced order methods for modeling and computational reduction, volume 9. Springer, 2014.

[44] Alfio Quarteroni, Gianluigi Rozza, and Andrea Manzoni. Certified reduced basis approximation for parametrized partial differential equations and applications. Journal of Mathematics in Industry, 1 (1):3, 2011.

[45] Clarence W Rowley. Model reduction for fluids, using balanced proper orthogonal decomposition. International Journal of Bifurcation and Chaos, 15(03):997-1013, 2005.

[46] Clarence W Rowley, Tim Colonius, and Richard M Murray. Model reduction for compressible flows using pod and galerkin projection. Physica D: Nonlinear Phenomena, 189(1):115-129, 2004.

[47] Peter J Schmid. Dynamic mode decomposition of numerical and experimental data. Journal of fluid mechanics, 656:5-28, 2010.

[48] Thomas W Sederberg and Scott R Parry. Free-form deformation of solid geometric models. ACM SIGGRAPH computer graphics, 20(4):151-160, 1986.

[49] Sirod Sirisup, George Em Karniadakis, Dongbin Xiu, and Ioannis G Kevrekidis. Equationfree/galerkin-free pod-assisted computation of incompressible flows. Journal of Computational Physics, 207(2):568-587, 2005.

[50] Lawrence Sirovich. Turbulence and the dynamics of coherent structures part i: coherent structures. Quarterly of applied mathematics, 45(3):561-571, 1987.

[51] PRaA Spalart and S1 Allmaras. A one-equation turbulence model for aerodynamic flows. In 30th aerospace sciences meeting and exhibit, page 439, 1992.

[52] James L Thomas and MD Salas. Far-field boundary conditions for transonic lifting solutions to the euler equations. AIAA journal, 24(7):1074-1080, 1986. 
[53] Mark G Turner, John A Reed, Robert Ryder, and Joseph P Veres. Multi-fidelity simulation of a turbofan engine with results zoomed into mini-maps for a zero-d cycle simulation. In $A S M E$ Turbo Expo 2004: Power for Land, Sea, and Air, pages 219-230. American Society of Mechanical Engineers, 2004.

[54] Massimiliano Vasile, Edmondo Minisci, Domenico Quagliarella, Marc Guénot, Ingrid Lepot, Caroline Sainvitu, Jordan Goblet, and Rajan Filomeno Coelho. Adaptive sampling strategies for nonintrusive pod-based surrogates. Engineering computations, 30(4):521-547, 2013.

[55] Zhu Wang, Imran Akhtar, Jeff Borggaard, and Traian Iliescu. Proper orthogonal decomposition closure models for turbulent flows: a numerical comparison. Computer Methods in Applied Mechanics and Engineering, 237:10-26, 2012.

[56] Jessie Weller, Simone Camarri, and Angelo Iollo. Feedback control by low-order modelling of the laminar flow past a bluff body. Journal of Fluid Mechanics, 634:405-418, 2009.

[57] Jessie Weller, Edoardo Lombardi, and Angelo Iollo. Robust model identification of actuated vortex wakes. Physica D: nonlinear phenomena, 238(4):416-427, 2009.

[58] Jessie Weller, Edoardo Lombardi, Michel Bergmann, and Angelo Iollo. Numerical methods for loworder modeling of fluid flows based on pod. International Journal for Numerical Methods in Fluids, 63(2):249-268, 2010.

[59] Karen Willcox and Jaime Peraire. Balanced model reduction via the proper orthogonal decomposition. AIAA journal, 40(11):2323-2330, 2002.

[60] Zhao Zhan, Wagdi G Habashi, and Marco Fossati. Local reduced-order modeling and iterative sampling for parametric analyses of aero-icing problems. AIAA Journal, 2015. 